\title{
Vascular flora of Lençóis Maranhenses National Park, Maranhão State, Brazil: checklist, floristic affinities and phytophysiognomies of restingas in the municipality of Barreirinhas
}

\author{
Misael Lira Rodrigues ${ }^{1 *}$ (1), Nara Furtado de Oliveira Mota ${ }^{2}$ (D), Pedro Lage Viana ${ }^{1,2}$ (1), Ana Kelly Koch ${ }^{3}$ (1) \\ and Ricardo de S. Secco ${ }^{2}$ (i)
}

Accepted: December 7, 2018

Received: May 21, 2019

\begin{abstract}
This study presents a floristic survey of the vascular plants of restingas of Lençóis Maranheses National Park, in the municipality of Barreirinhas, Maranhão, Brazil, including descriptions of the principal phytophysiognomies and similarity analyses including other restinga areas in North and Northeastern Brazil. Samples from the study area deposited in the herbaria MG, IAN and MAR were inventoried and fieldwork for the collection of additional botanical samples was undertaken between September 2015 and August 2017. Unweighted pair group method with arithmetic mean (UPGMA) using Jaccard index was used to calculate the similarity among floras of the restingas of the states of Pará, Piauí, Ceará and Maranhão. A total of 289 species in 189 genera and 73 families was recorded, including 56 new occurence records for the state of Maranhão. The richest families were Poaceae, Cyperaceae, Fabaceae, Rubiaceae, Eriocaulaceae and Myrtaceae. Similarity indexes revealed low similarity among the selected areas, suggesting high floristic identity for each area, as well as possible collection bias among the areas.
\end{abstract}

Keywords: dunes, floristics, new occurrence records, seed plants, white sand vegetation

\section{Introduction}

Maranhão state is situated in the Northeastern region of Brazil and occupies an area of more than $330,000 \mathrm{~km}^{2}$. It is situated within an area of transition between three different biomes: Cerrado, Amazon and Caatinga (Aragão \& Conceição 2008). Among the different vegetation types found in this state, the restinga is a coastal scrub occurring always along the sandy coast in the Amazon and Atlantic Forest biomes (Maranhão 2002).

In the northeastern region of Maranhão state there is an extensive area dominated by sandy sedimentary eolic deposits occupying approximately $18,000 \mathrm{~km}^{2}$ concentrated in the coastal region (Bandeira 2013). This area is situated in the transition between the three biomes found in the state, forming a mosaic of environments associated with sandy ecosystems, considered unique in Brazil (Saraiva \& Fernandes-Pinto 2007). According to the Brazilian vegetation map (IBGE 2012), this area is dominated by pioneering formations with the occurence of vegetation with a marine influence (restingas, mangroves), associated with savannas and tension zones between savanna and seasonal forest (Castro \& Piorski 2002). Situated in this region, the Lençóis Maranhenses National Park (PNLM) is considered a 'tropical desert' constituted by extensive dunes

\footnotetext{
1 Programa de Pós-Graduação em Ciências Biológicas, Museu Paraense Emílio Goeldi/Universidade Federal Rural da Amazônia, 66077-530, Belém, PA, Brazil

2 Coordenação de Botânica, Museu Paraense Emílio Goeldi, 66077-530, Belém, PA, Brazil

3 Departamento de Botânica e Ecologia, Instituto de Biociências, Universidade Federal de Mato Grosso, 79060-900, Cuiabá, MT, Brazil
}

* Corresponding author: mlirarodrigues@gmail.com 
and thousands of freshwater lakes (Damme \& Dumont 2010), of notable scenic beauty. According to MoschiniCarlos et al. (2008), the Lençóis Maranhenses correspond to a single series of dunes that extend from the Gulf of Maranhão up to the Parnaíba river, begining at the coast and advancing towards the continent for around $50 \mathrm{~km}$, influenced by river courses and the sea.

A total of 3064 species of Angiosperm are attributed to Maranhão state, ranking as 19th in floristic richness amonst the states of Brazil (Flora do Brasil 2020 2018). Despite including part of three major Brazilian biomes, the flora documented in Maranhão corresponds to only a little more than $9 \%$ of the Brazilian flora (Flora do Brasil 2020 2018). Important studies on coastal vegetation in the state of Maranhão were carried out, such as those of Araújo et al. (2016) and Lima \& Almeida Júnior (2018). However, collection efforts are necessary to enhance the extant collections and in order to produce more precise evaluation of the state's floristic composition.

Floristic inventories were carried out in some restinga areas in Maranhão (Serra et al. 2016; Almeida Júnior et al. 2017; Lima \& Almeida Júnior 2018), however, few studies on the flora have been developed specifically for the PNLM, apart from some scattered specimens deposited in herbaria, and the study by Moschini-Carlos et al. (2008), that describe the morphometric, physical and chemical variables of the ponds in PNLM, with emphasis on the planktonic community. Despite the importance of these studies, knowledge of the park's flora remains elementary.

The objective of this paper is to complete a first survey of the vascular flora in the Lençóis Maranhenses National Park, municipality of Barreirinhas, including a description of the principal phytophysiognomies as well as to carry out floristic comparisons with other restingas in the North and Northeastern regions of Brazil.

\section{Materials and methods}

\section{Study area}

This study was carried out in the Lençóis Maranhenses National Park (PLNM) located on the eastern coast of the state, $2^{\circ} 38^{\prime} 24^{\prime \prime S} 42^{\circ} 50^{\prime} 48^{\prime \prime} \mathrm{W}$ (Fig. 1). The PNLM, established $2^{\text {nd }}$ June 1981 by decree number 86,060 (ICMBIO 2016), consists of around 155 thousand hectares dominated by areas of restinga, encompassing the municipalities of Primeira Cruz, Santo Amaro and Barreirinhas. The sampling was restricted to the municipality of Barreirinhas, within

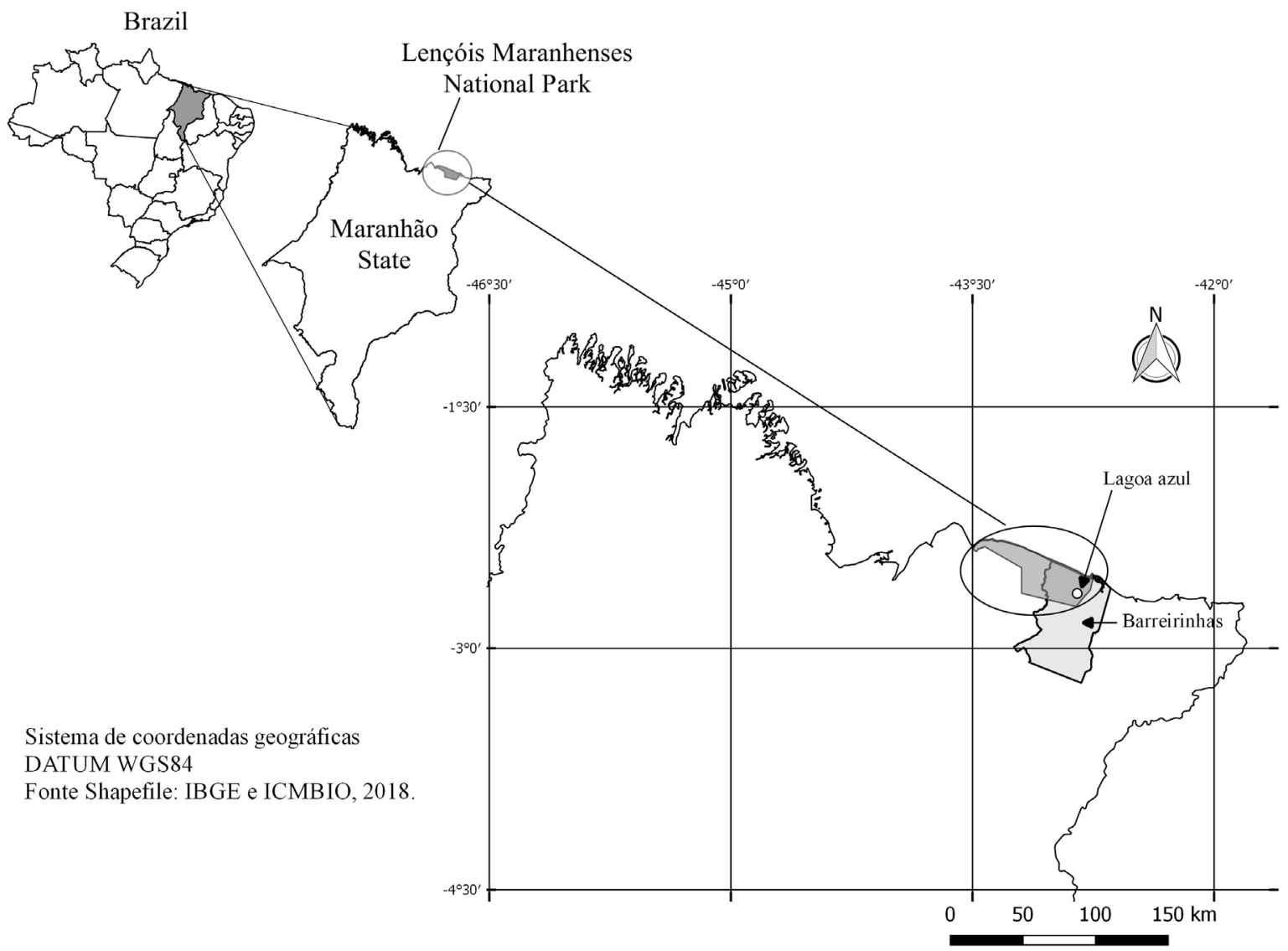

Figure 1. Map showing the study area: municipality of Barreirinhas in the Lençóis Maranhenses National Park. 
the PNLM, covering 302 hectares. The average annual temperature of the region is $28.5^{\circ} \mathrm{C}$, with most frequent precipitation in February and May, and a dry season from June to January (Miranda et al. 2012; ICMBIO 2016). Considered a transition zone between the semiarid climate and equatorial humidity, the landscape of the Lençóis Maranhenses is shaped by rivers carrying sediments in the direction of the coast, causing the formation of the restingas in these zones. These landscapes are characteristic to the region and consist of white dunes populated by a particular vegetation (Moschini-Carlos \& Pompêo 2016). This vegetation is composed of a mosaic of phytophysiognomies, generally dominated by coastal environments such as restingas and mangroves. Considering its peculiar phytophysiognomies, a brief description of the types found within the study area is proposed in this work, adapted from Bastos (1995) and Bastos et al. (2003) for the restingas of coastal Pará state.

\section{Botanical collections and herborization}

Seven expeditions for the collection of botanical material, each lasting at least five days, were carried out from September 2015 to December 2017, considering dry and wet periods. Collections were restricted to vascular plants, seeking to sample all of the habitats of the study area. Only fertile samples were collected. The samples were herborized according to appropriate techniques (Fidalgo \& Bononi 1989; IBGE 2012; Walter \& Fagg 2015) and incorporated into the MG Herbarium (herbarium acronyms according to Thiers 2009).

\section{Herbarium survey}

Parallel to the fieldwork, collections of the herbaria MG, IAN, and MAR were studied and inventoried, seeking samples from the municipality of Barreirinhas with the aim of adding them to the PNLM database that was used to generate the species list presented in this work.

\section{Identification of botanical samples}

The collections studied were identified with reference to the relevant taxonomical bibliography; comparison with samples previously deposited in the Herbarium MG and identified by specialists; and, when necessary, duplicates were sent to specialists to confirm the identifications.

\section{Preparation of species list}

Based on the specimen database from the study area, a list of vascular plants was generated, alphabetically organized according to family, indicating, for each species: voucher (collector + collector number + herbarium); distribution in Brazil by biome based on the Flora of Brazil online 2020
(FLORA DO BRASIL 2020 2018); habitat, based on the description of the vegetation proposed here, and habit, based on the categories followed by Flora do Brasil 2020 (2018).

The angiosperm list follows the APG IV (2016) classification system, with exception of Cordiaceae and Heliotropiaceae kept apart from Boraginaceae s.l. (BWG 2016) and Passifloraceae s.s. For ferns, Smith et al. (2006) and Rothfels et al. (2012) were followed, while for the lycophytes, Kramer \& Green (1990).

The species list of the PNLM was compared with the threatened species list for the flora of Brazil online (MMA 2014), available at the National Centre of Conservation of Flora, for the confirmation of the occurrence of species threatened with extinction in the study area (CNCFLORA 2018).

\section{Similarity analysis}

The floristic composition of the area studied was compared with surveys completed in areas of restinga Northern and Northeastern Brazil: data from two areas in the state of Pará, in the municipalities of Algodoal/ Maiandeua and Crispim/Marapanim (Amaral et al. 2008) were compiled and merged into a list for Pará state restingas; a first compilation of the vascular flora of the restingas in Maranhão state, based on herbarium survey (Almeida Júnior et al. 2017); restingas in Piauí state, in the municipalities of Ilha Grande, Parnaíba, Luís Correia and Cajueiro da Praia (Santos-Filho et al. 2015); and a checklist of the restingas in the state of Ceará (Santos-Filho et al. 2011). All of these listings had synonymy adjusted manually according to consultation of the Flora of Brazil 2020 database. Imprecisely identified (cf. or aff.) or material not identified to species level was excluded from the analysis. The species lists of the compared areas are available as supplementary material. The similarity analysis was completed utilizing the Jaccard index (Sneath \& Sokal 1973), by the non-weighted pair method with Arithmetic Mean (UPGMA).

\section{Results}

\section{Checklist}

The specimens compiled from the studied herbaria comprised 59 herbarium sheets from MG, 154 from IAN and 347 from MAR. The fieldwork contributed 525 samples, which are deposited in MG, thus totalling 1085 materials from the Barreirinhas municipality.

The analysis of these samples revealed the occurrence of 289 species, consisting of 286 angiosperms, two ferns and one lycophyte. Within the angiosperms, $63 \%$ of the species are Eudicots ( 178 species), 36\% are Monocots (105 species) and $1 \%$ of the group belongs to the Magnoliids (three species, Tab. 1). 
Vascular flora of Lençóis Maranhenses National Park, Maranhão State, Brazil: checklist, floristic affinities and phytophysiognomies of restingas in the municipality of Barreirinhas

Table 1. Checklist of the vascular plant species in the Lençóis Maranhenses National Park Barreirinhas, Maranhão state, Brazil. Abbreviations for collector names: Mo = N.F.O. Mota; $S=$ O. Silva; Marc = M.C.F.V. dos Santos; Mt = K.L.Martins; M = R.C. de Mendonça; B = J.M.Brito; $\mathrm{Ca}=$ G.C.A Carvalho; La = D.M.A Lacerda; Sa = S.M. Santos; Ex = E.F.B. de Carvalho; Cb = C. Cabral; Me $=$ F.N.Mendes; $\mathrm{Rm}=$ M.C.Ramos; $\mathrm{Rh}=\mathrm{R}$. Henriques; $\mathrm{O}=\mathrm{R} . \mathrm{P}$. Orlandi; Bo = C.B. de A. Bohrer; $\mathrm{R}=$ M.L. Rodrigues. Biomes: Amz = Amazon rainforest; $\mathrm{Atl}=$ Atlantic rainforest $; \mathrm{Caa}=\mathrm{Caatinga} ; \mathrm{Cer}=\mathrm{Cerrado} ; \mathrm{Pmp}=\mathrm{Pampa} ; \mathrm{Pnt}=$ Pantanal. Habitats: Hv = Halophytic Vegetation; Rfi = Restinga fields; Sr = Shrubby Restinga; Sc = Scrub; Il = Interdunal lagoons; Rfo = Restinga forest; $\mathrm{Cf}=$ Cocais forest; $\mathrm{Gf}=$ Gallery forest and Mangroves. Habits: $\mathrm{He}=\mathrm{Herb} ; \mathrm{Sh}=\mathrm{Shrub} ; \mathrm{Tr}=\mathrm{Tree} ; \mathrm{Vi}=$ Vine. First records for the state of Maranhão are assigned with + , endangered species with ${ }^{*}$.

\begin{tabular}{|c|c|c|c|c|}
\hline Family/ Species & Voucher & Occurence by biome & Habitat & Habit \\
\hline \multicolumn{5}{|l|}{ FERNS AND LYCOPHYTES } \\
\hline \multicolumn{5}{|l|}{ DENNSTAEDTIACEAE } \\
\hline Pteridium arachnoideum (Kaulf.) Maxon + & R 40 (MG-225203) & Amz, Caa, Cer, Atl, Pmp, Pnt & $\mathrm{Sr}$ & $\mathrm{He}$ \\
\hline \multicolumn{5}{|l|}{ LYCOPODIACEAE } \\
\hline Palhinhaea cernua (L.) Franco \& Vasc. & Mo 3120 (MG-214749) & Amz, Cer, Atl, Pmp, Pnt & Rfi & $\mathrm{He}$ \\
\hline \multicolumn{5}{|l|}{ SCHIZAEACEAE } \\
\hline Actinostachys pennula (Sw.) Hook. + & Mo 3197 (MG-214826) & Amz, Cer, Atl & Rfi & $\mathrm{He}$ \\
\hline \multicolumn{5}{|l|}{ ANGIOSPERMS } \\
\hline \multicolumn{5}{|l|}{ ACANTHACEAE } \\
\hline Thyrsacanthus secundus (Leonard) A.Cortês \& Rapini & R 243 (MG-228215) & $\mathrm{Amz}$ & $\mathrm{Sr}$ & Sh \\
\hline \multicolumn{5}{|l|}{ ALISMATACEAE } \\
\hline Helanthium tenellum (Mart.) Britton & R 12 (MG-225175); Cb 319 (MAR-2714) & Amz, Caa, Cer, Atl & Rfi & $\mathrm{He}$ \\
\hline \multicolumn{5}{|l|}{ ALSTROEMERIACEAE } \\
\hline Bomarea edulis (Tussac) Herb. & B 48 (MG-154873) & Amz, Caa, Cer, Atl, Pnt & Sc & $\mathrm{Vi}$ \\
\hline \multicolumn{5}{|l|}{ AMARANTHACEAE } \\
\hline Blutaparon portulacoides (A.St.-Hil.) Mears & R 90 (MG-225253) & Cer, Atl & $\mathrm{Hv}$ & $\mathrm{He}$ \\
\hline Gomphrena sp. & Mo 3233 (MG-214862) & & Rfi & $\mathrm{He}$ \\
\hline \multicolumn{5}{|l|}{ AMARYLLIDACEAE } \\
\hline Zephyranthes cearensis (Herb.) Baker & S 42 (IAN-181074); Ca 25 (IAN-185249) & Caa, Cer & $\mathrm{Sr}$ & $\mathrm{He}$ \\
\hline \multicolumn{5}{|l|}{ ANACARDIACEAE } \\
\hline Anacardium occidentale L. & $\begin{array}{l}\text { B } 16 \text { (MG-154815); R } 01 \text { (MG-225164); } \\
\text { Sa } 15 \text { (MAR-5970) }\end{array}$ & Amz, Caa, Cer, Atl, Pmp, Pnt & Sc & $\operatorname{Tr}$ \\
\hline Tapirira guianensis Aubl. & R 60 (MG-225223) & Amz, Caa, Cer, Atl, Pmp, Pnt & $\mathrm{Sr}$ & $\operatorname{Tr}$ \\
\hline \multicolumn{5}{|l|}{ ANNONACEAE } \\
\hline Duguetia echinophora R.E.Fr. & S 25 (MG-178602); R 155 (MG-225737) & Amz, Cer & $\mathrm{Sr}$ & $\operatorname{Tr}$ \\
\hline Xylopia sericea A.St.-Hil. & В 27 (MG-154822) & Amz, Cer, Atl & Rs & $\mathrm{Sh} / \mathrm{Tr}$ \\
\hline \multicolumn{5}{|l|}{ APOCYNACEAE } \\
\hline Allamanda blanchetii A.DC. & Bo 45 (MG-154824), Bo 46 (MG-154825) & Caa, Cer & $\mathrm{Sr}$ & Sh \\
\hline Blepharodon pictum (Vahl) W.D.Stevens & S 75 (MAR-6162); Sa s.n (MAR-6606). & Amz, Caa, Cer, Atl & $\mathrm{Sr}$ & $\mathrm{Vi}$ \\
\hline Ditassa banksii R.Br. ex Schult. & Sa s.n. (MAR-6605) & Atl & $\mathrm{Sr}$ & Sh \\
\hline Hancornia speciosa Gomes & B 29 (MG-154823) & Amz, Caa, Cer, Atl & $\mathrm{Sr}$ & $\operatorname{Tr}$ \\
\hline Himatanthus articulatus (Vahl) Woodson + & $\begin{array}{l}\text { R } 23 \text { (MG-225186), R } 180 \text { (MG-225762); } \\
\text { Ca } 23 \text { (IAN-185247); S } 17 \text { (MAR-6120) }\end{array}$ & $\mathrm{Amz}, \mathrm{Cer}$ & $\mathrm{Sr}$ & $\operatorname{Tr}$ \\
\hline Mandevilla hirsuta (A.Rich.) K.Schum. & R 242 (MG-228214); S 86 (MAR6112) & Amz, Caa, Cer, Atl & $\mathrm{Sr}$ & $\mathrm{Vi}$ \\
\hline $\begin{array}{c}\text { Mandevilla scabra (Hoffmanns. ex Roem. \& Schult.) } \\
\text { K.Schum. }\end{array}$ & $\begin{array}{c}\text { Mo } 3106 \text { (MG-214735; R } 120 \text { (MG-225702), } \\
\text { R } 122 \text { (MG-225704) }\end{array}$ & Amz, Caa, Cer, Atl & Sr/Rfi & $\mathrm{Vi}$ \\
\hline Secondatia densiflora A.DC. & Sa 22 (IAN-187629) & Amz, Caa, Cer, Atl & $\mathrm{Sr}$ & $\mathrm{Vi}$ \\
\hline \multicolumn{5}{|l|}{ ARECACEAE } \\
\hline Astrocaryum chambira Burret & Sa s.n (MAR-6100) & $\mathrm{Amz}$ & $\mathrm{Sr} / \mathrm{Cf}$ & $\operatorname{Tr}$ \\
\hline Copernicia prunifera (Mill.) H.E.Moore & R 57 (MG-225220); Sa s.n. (MAR-6099) & Caa, Cer & $\mathrm{Sr} / \mathrm{Cf}$ & $\operatorname{Tr}$ \\
\hline \multicolumn{5}{|l|}{ ASTERACEAE } \\
\hline Lepidaploa arenaria (Mart. ex DC) H.Rob. + & $\begin{array}{c}\text { Mo } 3213 \text { (MG-214842); Sa s.n. (MAR-6092); } \\
\text { S } 771 \text { (MAR-6042) }\end{array}$ & Amz, Atl & Sr & Sh \\
\hline Stilpnopappus cearensis Huber + & Mo 3232 (MG-214861) & Caa & $\mathrm{Sr}$ & $\mathrm{He}$ \\
\hline Tilesia baccata (L.F.) Pruski & $\begin{array}{l}\text { S } 31 \text { (MG-178149); R } 216 \text { (MG-225798); } \\
\text { Mo } 3226 \text { (MG-214855) }\end{array}$ & $\begin{array}{l}\text { Amz, Caa, Cer, } \\
\text { Mata Atlêntica }\end{array}$ & $\mathrm{Sr}$ & Sh \\
\hline \multicolumn{5}{|l|}{ BIGNONIACEAE } \\
\hline Adenocalymma validum L.G.Lohmann & Rm 68 (MG-174775) & Amz, Caa, Atl & Rfo & $\mathrm{Vi}$ \\
\hline Cuspidaria lateriflora (Mart.) DC. & S 39 (MG-178157) & Amz, Caa, Cer, Atl & Sr/Rfo & $\mathrm{Vi}$ \\
\hline Fridericia dispar (Bureau ex K. Schum.) L.G.Lohmann + & S 38 (MG-178156) & Caa, Cer & $\mathrm{Sr}$ & $\mathrm{Sh} / \mathrm{Vi}$ \\
\hline
\end{tabular}




\section{Misael Lira Rodrigues, Nara Furtado de Oliveira Mota, Pedro Lage Viana, Ana Kelly Koch and Ricardo de S. Secco}

Table 1. Cont.

\begin{tabular}{|c|c|c|c|c|}
\hline Family/Species & Voucher & Occurence by biome & Habitat & Habit \\
\hline Tabebuia roseoalba (Ridl.) Sandwith & R 290 (MG-228262) & Caa, Cer, Atl & $\mathrm{Sr}$ & $\operatorname{Tr}$ \\
\hline \multicolumn{5}{|l|}{ BURMANNIACEAE } \\
\hline Burmania capitata (Walter ex J.F.Gmel) Mart. & Mo 3174 (MG-214803) & Amz, Cer, Atl & Rfi & $\mathrm{He}$ \\
\hline \multicolumn{5}{|l|}{ BURSERACEAE } \\
\hline Protium heptaphyllum subsp. ulei (Swart) Daly & $\begin{array}{l}\text { Mo } 3069 \text { (MG-214698); R } 79 \text { (MG-225242); } \\
\text { R } 81 \text { (MG-225244); R } 281 \text { (MG-228253); } \\
\text { Sa } 13 \text { (IAN-187623); S } 06 \text { (MAR-6044) }\end{array}$ & $\mathrm{Amz}$ & Rfo & $\operatorname{Tr}$ \\
\hline \multicolumn{5}{|l|}{ CACTACEAE } \\
\hline $\begin{array}{l}\text { Pilosocereus catingicola subsp. Salvadorensis } \\
\text { (Werderm.) Zappi + }\end{array}$ & R 55 (MG-225218) & Caa, Atl & Rfo & $\mathrm{Tr} / \mathrm{Sh}$ \\
\hline \multicolumn{5}{|l|}{ CALOPHYLLACEAE } \\
\hline Calophyllum brasiliense Cambess. + & R 183 (MG-225765) & Amz, Caa, Cer, Atl & $\mathrm{Sr}$ & $\operatorname{Tr}$ \\
\hline \multicolumn{5}{|l|}{ CARYOCARACEAE } \\
\hline Caryocar brasiliense Cambess. + & Sa 08 (MAR-6078) & Amz, Caa, Cer, Atl & Rfo & $\operatorname{Tr}$ \\
\hline \multicolumn{5}{|l|}{ CELASTRACEAE } \\
\hline Monteverdia erythroxyla (Reissek) Biral & $\begin{array}{l}\text { Mo } 3059 \text { (MG-214688), Mo } 3163 \text { (MG- } \\
\text { 214792); R } 42 \text { (MG-225205), R } 135 \text { (MG- } \\
\text { 225717), R } 157 \text { (MG-225739) }\end{array}$ & Caa, Cer, Atl & $\mathrm{Sr}$ & $\mathrm{Tr} / \mathrm{Sh}$ \\
\hline \multicolumn{5}{|l|}{ CHRYSOBALANACEAE } \\
\hline Chrysobalanus icaco L. & $\begin{array}{c}\text { Mo } 3126 \text { (MG-214755); R } 31 \text { (MG-225194), } \\
\text { R } 84 \text { (MG-225247), R } 127 \text { (MG-225709), R } \\
201 \text { (MG-225783); Sa } 05 \text { (MAR-5962), Sa } 25 \\
\text { (MAR-5966); S } 01 \text { (MAR-6114) }\end{array}$ & Amz, Atl & $\mathrm{Sc} / \mathrm{Sr}$ & $\mathrm{Sh} / \mathrm{Tr}$ \\
\hline Couepia guianensis subsp. glandulosa (Miq.) Prance & S 41 (IAN-181362) & $\mathrm{Amz}$ & $\mathrm{Sr}$ & $\operatorname{Tr}$ \\
\hline Hirtella ciliata Mart. \& Zucc. & La s.n. (MAR-7071) & Amz, Caa, Cer, Atl & $\mathrm{Sr}$ & $\mathrm{Sh} / \mathrm{Tr}$ \\
\hline \multicolumn{5}{|l|}{ CLUSIACEAE } \\
\hline Clusia grandiflora Splitg. & $\begin{array}{l}\text { Mo } 3103 \text { (MG-214732); R } 56 \text { (MG-225219), } \\
\text { R } 133 \text { (MG-225715); Sa } 20 \text { (IAN-188976); S } \\
80 \text { (MAR-6167) }\end{array}$ & $\mathrm{Amz}$ & $\mathrm{Sr}$ & $\operatorname{Tr}$ \\
\hline \multicolumn{5}{|l|}{ COMBRETACEAE } \\
\hline Conocarpus erectus L. & $\begin{array}{l}\text { Mo3151 (MG-214780); R } 91 \text { (MG-225254), R } \\
221 \text { (MG-225803), R } 314 \text { (MG-228286); }\end{array}$ & Amz, Atl & $\mathrm{Hv}$ & Sh \\
\hline \multicolumn{5}{|l|}{ CONNARACEAE } \\
\hline Connarus favosus Planch. & B 57 (MG-154874) & $\mathrm{Amz}, \mathrm{Caa}$ & Rfo & $\mathrm{Sh} / \mathrm{Vi}$ \\
\hline \multicolumn{5}{|l|}{ CONVOLVULACEAE } \\
\hline Ipomoea blanchetii Choisy & Mo 3072 (MG-214701) & Amz, Caa, Cer, Atl & $\mathrm{Sr}$ & $\mathrm{Vi}$ \\
\hline Ipomoea brasiliana (Choisy) Meisn. & $\begin{array}{l}\text { Mo } 3055 \text { (MG-214684); R } 165 \text { (MG-225747); } \\
\text { S } 22 \text { (MG-178138); Ca } 49 \text { (IAN-185224) }\end{array}$ & Caa, Cer & $\mathrm{Sr}$ & $\mathrm{Vi}$ \\
\hline Ipomoea pes-caprae (L.) R.Br. & R 66 (MG-225229) & Amz, Atl & $\mathrm{Sr} / \mathrm{Sc}$ & $\mathrm{He} / \mathrm{Vi}$ \\
\hline Ipomoea sp. & S 49 (IAN-181346) & - & $\mathrm{Sr}$ & $\mathrm{Vi}$ \\
\hline \multicolumn{5}{|l|}{ CYPERACEAE } \\
\hline Bulbostylis cf. capillaris (L.) C.B.Clarcke & Mo 3071 (MG-214700) & Amz, Caa, Cer, Atl, Pmp, Pnt & $\mathrm{Sr}$ & $\mathrm{He}$ \\
\hline Bulbostylis conifera (Kunth) C.B.Clarcke & $\begin{array}{c}\text { Mo } 3081 \text { (MG-214710), Mo } 3122 \text { (MG- } \\
\text { 214751); R } 139 \text { (MG-225721), R } 208 \text { (MG- } \\
\text { 225790) }\end{array}$ & Amz, Caa, Cer, Atl & Rfi & $\mathrm{He}$ \\
\hline Bulbostylis junciformis (Kunth.) C.B.Clarke & $\begin{array}{c}\text { R } 137 \text { (MG-225719), R } 173 \text { (MG-225755), R } \\
204 \text { (MG-225786) }\end{array}$ & Amz, Caa, Cer, Atl, Pmp, Pnt & Rfi & $\mathrm{He}$ \\
\hline Bulbostylis lagoensis (Boeck) Prata \& M.G.Lopez + & Mo 3051 (MG-214680) & Cer & Rfi & $\mathrm{He}$ \\
\hline Bulbostylis lanata (Kunth) Lindm. & $\begin{array}{c}\text { Mo } 3102 \text { (MG-214731); R } 30 \text { (MG-225193), } \\
\text { R } 82 \text { (225245), R } 143 \text { (MG-225725), R } 145 \\
\text { (MG-225727) }\end{array}$ & Amz, Cer, Atl & Sr & $\mathrm{He}$ \\
\hline Cyperus articulatus L. & Mo 3025 (MG-214654) & Amz, Caa, Mata Atlêntica & $\mathrm{Hv}$ & $\mathrm{He}$ \\
\hline Cyperus compressus L. & Mo 3032 (MG-214661) & Amz, Caa, Cer, Atl, Pnt & Rfi & $\mathrm{He}$ \\
\hline Cyperus crassipes Vahl & R 197 (MG-225779), R 308 (MG-228280) & Caa, Atl & $\mathrm{Sc}$ & $\mathrm{He}$ \\
\hline Cyperus haspan L. & $\begin{array}{c}\text { Mo } 3124 \text { (MG-214753), Mo } 3166 \text { (MG- } \\
\text { 214795); R } 260 \text { (MG-228232), R } 311 \text { (MG- } \\
\text { 228283) }\end{array}$ & Amz, Caa, Cer, Atl, Pmp, Pnt & Rfi & $\mathrm{He}$ \\
\hline
\end{tabular}


Vascular flora of Lençóis Maranhenses National Park, Maranhão State, Brazil: checklist, floristic affinities and phytophysiognomies of restingas in the municipality of Barreirinhas

Table 1. Cont.

\begin{tabular}{|c|c|c|c|c|}
\hline Family/ Species & Voucher & Occurence by biome & Habitat & Habit \\
\hline Cyperus ligularis L. & $\begin{array}{c}\text { Mo } 3022 \text { (MG-214651); R } 195 \text { (MG-225777), } \\
\text { R } 310 \text { (MG-228282) }\end{array}$ & Amz, Caa, Cer, Atl & Sr & $\mathrm{He}$ \\
\hline Cyperus obtusatus (J.Presl. \& C.Presl.) Mattf. \& Kük. & $\begin{array}{l}\text { Mo } 3030 \text { (MG-214659); R } 77 \text { (MG-225240), } \\
\text { R } 198 \text { (MG-225780), R } 228 \text { (MG-225810) }\end{array}$ & Amz, Caa, Cer, Atl, Pmp, Pnt & $\mathrm{Hv}$ & $\mathrm{He}$ \\
\hline Cyperus odoratus L. & Mo 3216 (MG-214845) & Amz, Caa, Cer, Atl, Pmp, Pnt & $\mathrm{Sr}$ & $\mathrm{He}$ \\
\hline Cyperus subsquarrosus (Muhl.) Bauters & Mo 3024 (MG-214653) & Amz, Caa, Cer, Atl, Pmp, Pnt & $\mathrm{Hv}$ & $\mathrm{He}$ \\
\hline Cyperus tenuispica Steud. + & Mo 3155 (MG-214784) & Amz, Cer & Rfi & $\mathrm{He}$ \\
\hline Eleocharis endounifascis Hinchliff \& Roalson & $\begin{array}{c}\text { Mo } 3127 \text { (MG-214756); R } 146 \text { (MG-225728), } \\
\text { R } 261 \text { (MG-228233) }\end{array}$ & Amz, Caa, Atl, Pmp & Rfi & $\mathrm{He}$ \\
\hline Eleocharis geniculata (L.) Roem \& Schult. & $\begin{array}{c}\text { Mo } 3029 \text { (MG-214658), Mo } 3035 \text { (MG- } \\
\text { 214664); R } 09 \text { (MG-225172), R } 76 \text { (MG- } \\
\text { 225239), R } 224 \text { (MG-225806), R } 317 \\
\text { (MG-228289) }\end{array}$ & Amz, Caa, Cer, Atl, Pmp, Pnt & $\mathrm{Hv} / \mathrm{Rfi}$ & $\mathrm{He}$ \\
\hline Eleocharis nana Kunth & Mo 3048 (MG-214677); R 17 (MG-225180) & Amz, Caa, Cer, Atl & Rfi & $\mathrm{He}$ \\
\hline Fimbristylis cymosa R.Br. & $\begin{array}{c}\text { Mo } 3019 \text { (MG-214648), Mo } 3144 \text { (MG- } \\
\text { 214773); R } 86 \text { (MG-225249), R } 227 \text { (MG- } \\
\text { 225809) }\end{array}$ & Amz, Caa, Cer, Atl & $\mathrm{Hv} / \mathrm{Rfi}$ & $\mathrm{He}$ \\
\hline Fimbristylis dichotoma (L.) Vahl & Mo 3148 (MG-214777); R 88 (MG-225251) & Amz, Caa, Cer, Atl, Pmp, Pnt & $\mathrm{Hv}$ & $\mathrm{He}$ \\
\hline Fimbristylis miliacea (L.) Vahl & R 168 (MG-225750) & Amz, Caa, Cer, Atl, Pmp, Pnt & Sr & $\mathrm{He}$ \\
\hline Fimbristylis spadicea (L.) Vahl & R 231 (MG-225813) & Amz, Caa, Cer, Atl & Sc & $\mathrm{He}$ \\
\hline Fuirena umbellata Rottb. & Mo 3185 (MG-214814) & Amz, Caa, Cer, Atl, Pmp, Pnt & Rfi & $\mathrm{He}$ \\
\hline Hypolytrum pulchrum (Rudge) H.Pfeiff + & Mo 3219 (MG-214848); R 172 (MG-225754) & Amz, Atl & $\mathrm{Sr}$ & $\mathrm{He}$ \\
\hline Lagenocarpus guianensis Lindl. ex Nees & Mo 3087 (MG-214716) & Amz, Caa, Cer, Atl & Sr/Rfi & $\mathrm{He}$ \\
\hline Lagenocarpus rigidus Nees & $\begin{array}{c}\text { Mo } 3184 \text { (MG-214813); R } 53 \text { (MG-225216), } \\
\text { R } 62 \text { (MG-225225) }\end{array}$ & Amz, Caa, Cer, Atl, Pnt & Sr/Rfi & $\mathrm{He}$ \\
\hline Lagenocarpus cf. sabanensis Gilly & R 147 (MG-225729) & Amz, Caa, Cer, Atl, Pnt & Sr/Rfi & $\mathrm{He}$ \\
\hline $\begin{array}{l}\text { Lagenocarpus verticillatus (Spreng.) T.Koyama \& } \\
\text { Maguire }\end{array}$ & $\begin{array}{l}\text { R } 50 \text { (MG-225213), R } 142 \text { (225724); } \\
\text { Mo } 3179 \text { (MG-214808) }\end{array}$ & Amz, Caa, Cer, Atl & Sr/Rfi & $\mathrm{He}$ \\
\hline Lagenocarpus sp. & R 148 (MG-225730), R 262 (MG-228234) & - & Sr/Rfi & $\mathrm{He}$ \\
\hline Rhynchospora barbata (Vahl) Kunth & $\begin{array}{c}\text { Mo } 3082 \text { (MG-214711), Mo } 3092 \text { (MG- } \\
\text { 214721); R } 26 \text { (MG-225189), R } 138 \\
\text { (MG-225720), R } 141 \text { (MG-225723), R } 259 \\
\text { (MG-228231) R } 296 \text { (MG-228268); Ca } 14 \\
\text { (IAN-185238) }\end{array}$ & Amz, Caa, Cer & Sr/Rfi & $\mathrm{He}$ \\
\hline Rhynchospora curvula Griseb. + & Mo 3169 (MG-214798) & Caa & Rfi & $\mathrm{He}$ \\
\hline Rhynchospora filiformis Vahl. + & R 144 (MG-225726) & $\mathrm{Amz}, \mathrm{Caa}, \mathrm{Cer}$ & Rfi & $\mathrm{He}$ \\
\hline Rhynchospora hirsuta (Vahl) Vahl & $\begin{array}{c}\text { Mo } 3093 \text { (MG-214722), Mo } 3114 \text { (MG- } \\
\text { 214743) }\end{array}$ & Amz, Cer & Rfi & $\mathrm{He}$ \\
\hline Rhynchospora holoschoenoides (Rich.) Herter & $\begin{array}{l}\text { R } 171 \text { (MG-225753); Mo } 3090 \text { (MG-214719), } \\
\text { Mo } 3218 \text { (MG-214847) }\end{array}$ & Amz, Caa, Cer, Atl, Pmp, Pnt & Sr/Rfi & $\mathrm{He}$ \\
\hline Rhynchospora tenuis Link. & $\begin{array}{l}\text { Mo } 3020 \text { (MG-214649), Mo } 3073 \text { (MG- } \\
\text { 214702), Mo } 3136 \text { (MG-214765); R } 20 \\
\text { (MG-225183) }\end{array}$ & Amz, Caa, Cer, Atl, Pmp, Pnt & Sc/Rfi & $\mathrm{He}$ \\
\hline Scleria martii (Nees) Steud. & $\begin{array}{c}\text { Mo } 3180 \text { (MG-214809); R } 52 \text { (MG-225215), } \\
\text { R } 149 \text { (MG-225731) }\end{array}$ & $\mathrm{Amz}, \mathrm{Cer}$ & Sr/Rfi & $\mathrm{He}$ \\
\hline Scleria microcarpa Nees & Mo 3101 (MG-214730); R 174 (MG-225756) & Amz, Caa, Cer, Atl, Pmp, Pnt & $\mathrm{Sr}$ & $\mathrm{He}$ \\
\hline \multicolumn{5}{|l|}{ DILLENIACEAE } \\
\hline Davilla cearensis Huber & $\begin{array}{c}\text { B } 41 \text { (MG-154872); R } 58 \text { (MG-225221); S } 08 \\
\text { (MAR-6048) }\end{array}$ & Amz, Cer, Atl & Sr & Vi \\
\hline Doliocarpus spraguei Cheesman + & $\begin{array}{c}\text { Mo } 3109 \text { (MG-214738); R } 234 \text { (MG-225816); } \\
\text { Sa } 05 \text { (IAN-188962) }\end{array}$ & $\operatorname{Amz}$ & Sr & $\mathrm{Sh} / \mathrm{Vi}$ \\
\hline \multicolumn{5}{|l|}{ DIOSCOREACEAE } \\
\hline Dioscorea multiflora Mart. ex Griseb. & Mo 3053 (MG-214682); R 160 (MG-225742) & Amz, Cer, Atl & $\mathrm{Sr}$ & $\mathrm{Vi}$ \\
\hline \multicolumn{5}{|l|}{ DROSERACEAE } \\
\hline Drosera sessilifolia A.St.-Hil. & $\begin{array}{l}\text { R } 249 \text { (MG-228221), R } 295 \text { (MG-228267); Sa } \\
\text { s/n (MAR-6613) }\end{array}$ & Amz, Caa, Cer, Atl, Pnt & Rfi & $\mathrm{He}$ \\
\hline ERIOCAULACEAE & & & & \\
\hline
\end{tabular}




\section{Misael Lira Rodrigues, Nara Furtado de Oliveira Mota, Pedro Lage Viana, Ana Kelly Koch and Ricardo de S. Secco}

Table 1. Cont.

\begin{tabular}{|c|c|c|c|c|}
\hline Family/Species & Voucher & Occurence by biome & Habitat & Habit \\
\hline Eriocaulon cinereum R.Br. + & $\begin{array}{l}\text { R } 13 \text { (MG-225176), R } 316 \text { (MG-228288); Mo } \\
3203 \text { (MG-214832) }\end{array}$ & Amz, Caa, Cer, Pnt & $\begin{array}{l}\text { Il/Sc/ } \\
\text { Rfi }\end{array}$ & $\mathrm{He}$ \\
\hline Eriocaulon setaceum L. & R 255 (MG-228227), R 272 (MG-228244) & Amz, Caa, Cer, Pnt & $\mathrm{Sr} / \mathrm{Il}$ & $\mathrm{He}$ \\
\hline Paepalanthus bifidus (Schard.) Kunth & Cb 11 (MAR-2634) & Amz, Caa, Cer, Atl & Rfi & $\mathrm{He}$ \\
\hline Paepalanthus polytrichoides Kunth + & Cb 8 (MAR-2633), Cb 14 (MAR-2635) & $\mathrm{Amz}$ & Rfi & $\mathrm{He}$ \\
\hline Paepalanthus sessiliflorus Mart. ex Körn. + & Mo 3217 (MG-214846) & $\mathrm{Amz}, \mathrm{Caa}, \mathrm{Cer}$ & Il & $\mathrm{He}$ \\
\hline Paepalanthus subtilis Miq. & Mo 3156 (MG-214785) & Amz, Caa, Cer, Atl & $\mathrm{Sr}$ & $\mathrm{He}$ \\
\hline $\begin{array}{c}\text { Syngonanthus cuyabenses (Bong.) Giul., Helsond \& } \\
\text { L.R.Parra }\end{array}$ & $\begin{array}{l}\text { R } 18 \text { (MG-225181), } \\
\text { R } 252 \text { (MG-228224) }\end{array}$ & Amz, Caa, Cer & Sr/Rfi & $\mathrm{He}$ \\
\hline Syngonanthus heteropeplus (Koern.) Ruhland & R 253 (MG-228225) & $\mathrm{Amz}, \mathrm{Cer}$ & $\mathrm{Sr}$ & $\mathrm{He}$ \\
\hline Syngonanthus philodicoides (Körn.) Ruhland + & R 293 (MG-228265) & Cer & Rfi & $\mathrm{He}$ \\
\hline Syngonanthus umbellatus (Lam.) Ruhland + & R 45 (MG-225208) & $\mathrm{Amz}, \mathrm{Cer}$ & $\mathrm{Sr}$ & $\mathrm{He}$ \\
\hline \multicolumn{5}{|l|}{ EUPHORBIACEAE } \\
\hline Croton pycnadenius Müll. Arg. & $\begin{array}{c}\text { R } 238 \text { (MG-25820); Mo } 3199 \text { (MG-214828); S } \\
57 \text { (IAN-181347), S } 68 \text { (IAN-181357) }\end{array}$ & Amz, Cer & Sr & $\mathrm{He}$ \\
\hline Croton sp. & R 163 (MG-225745) & - & $\mathrm{Sr}$ & $\operatorname{Tr}$ \\
\hline Dalechampia scandens L. & R 193 (MG-225775) & Amz, Caa, Cer, Atl, Pnt & $\mathrm{Sr}$ & $\mathrm{Vi}$ \\
\hline Euphorbia bahiensis (Klotzsch \& Garcke) Boiss & Mo 3210 (MG-214839) & Atl & $\mathrm{Sr}$ & $\mathrm{He}$ \\
\hline Mabea pohliana (Benth.) Müll.Arg. & R 239 (MG-225821) & Amz, Cer & $\mathrm{Sr}$ & $\mathrm{Sh} / \mathrm{Tr}$ \\
\hline Microstachys corniculata (Vahl) Griseb. & Mo 3140 (MG-214769) & Amz, Caa, Cer, Atl & Rfi & $\mathrm{He}$ \\
\hline \multicolumn{5}{|l|}{ FABACEAE } \\
\hline Abarema cochleata (Willd.) Barnabey \& J.W.Grimes & $\begin{array}{l}\text { R } 03 \text { (MG-225166); Mo } 3228 \text { (MG-214857); } \\
\text { Sa } 21 \text { (MAR-5968); Ex } 77 \text { (MAR-3243) }\end{array}$ & $\mathrm{Amz}$ & Sr/Rfo & $\operatorname{Tr}$ \\
\hline Aeschynomene brevipes Benth. & $\begin{array}{c}\text { Mo } 3205 \text { (MG-214834); R } 309 \text { (MG-228281); } \\
\text { Ca } 55 \text { (IAN-185220) }\end{array}$ & $\mathrm{Amz}, \mathrm{Caa}, \mathrm{Cer}$ & Sr/Rfi & $\mathrm{Sh} / \mathrm{He}$ \\
\hline Andira vermifuga (Mart.) Benth. & R 237 (MG-225819); Mo 3204 (MG-214833) & Amz, Caa, Cer, Atl & Rfo & $\operatorname{Tr}$ \\
\hline Bauhinia cf. dubia G.Don. & $\begin{array}{c}\text { Mo } 3237 \text { (MG-214866); S } 36 \text { (MG-178154); B } \\
35 \text { (MG-154868) }\end{array}$ & Amz, Cer & $\mathrm{Sr}$ & Sh \\
\hline Calliandra dysantha Benth. + & $\begin{array}{l}\text { R } 192 \text { (MG-225774), R } 279 \text { (MG-228251); } \\
\text { Mo } 3049 \text { (MG-214678); S } 67 \text { (MG-181356) }\end{array}$ & Caa, Cer & $\mathrm{Sr}$ & $\mathrm{Sh}$ \\
\hline Calliandra sessilis Benth. & B 67 (MG-154877) & $\mathrm{Amz}, \mathrm{Caa}, \mathrm{Cer}$ & $\mathrm{Sr}$ & $\operatorname{Tr}$ \\
\hline Centrosema brasilianum (L.) Benth. & S 32 (MG-178150); Sa 11 (MG-188969) & Amz, Caa, Cer, Atl, Pnt & $\mathrm{Sr}$ & Vi \\
\hline Centrosema pascuorum Mart. ex Benth. + & Mo 3130 (MG-214759) & Amz, Caa, Cer, Atl, Pnt & $\mathrm{Sr}$ & $\mathrm{Vi}$ \\
\hline Chamaecrista flexuosa (L.) Green & Mo 3206 (MG-214835); R 05 (MG-225168) & Amz, Caa, Cer, Atl, Pmp, Pnt & $\mathrm{Sr} / \mathrm{Sc}$ & $\mathrm{Sh} / \mathrm{He}$ \\
\hline Chamaecrista ramosa (Vogel) H.S.Irwin \& Barneby & $\begin{array}{c}\text { O } 648 \text { (MG-136967); Mo } 3123 \text { (MG-214752); } \\
\text { R } 33 \text { (MG-225196), R } 116 \text { (MG-225698) R } \\
124 \text { (MG-225706), R } 186 \text { (MG-225768); S } \\
50 \text { (IAN-181363); Sa } 07 \text { (MAR-5967); Ca } 05 \\
\text { (MAR-6590) }\end{array}$ & Amz, Caa, Cer, Atl, Pnt & Sr/Rfi & Sh \\
\hline Clitoria stipularis Benth. & Mo 3149 (MG-214778) & $\mathrm{Amz}, \mathrm{Caa}$ & Rfi & $\begin{array}{l}\mathrm{Sh} / \\
\mathrm{He} / \mathrm{Vi}\end{array}$ \\
\hline Copaifera martii Hayne & $\begin{array}{l}\text { Mo } 3060 \text { (MG-214689); S } 63 \text { (MG-154829); } \\
\text { R } 61 \text { (MG-225224), R } 154 \text { (MG-225736); Ca } \\
\text { s.n. (IAN-185276); S } 21 \text { (MAR-6055) }\end{array}$ & Amz, Caa, Cer & Sr/Rfo & $\mathrm{Sh} / \mathrm{Tr}$ \\
\hline Desmodium barbatum (L.) Benth. & Mo 3194 (MG-214823) & Amz, Caa, Cer, Atl, Pmp, Pnt & $\mathrm{Sr}$ & $\mathrm{He}$ \\
\hline Diplotropis sp. & S 23 (MG-178140) & - & $\mathrm{Sr}$ & $\operatorname{Tr}$ \\
\hline Dimorphandra gardneriana Tul. & B 59 (MG-154827) & Caa, Cer & Rfo & $\operatorname{Tr}$ \\
\hline Dioclea cf. virgata (Rich.) Amshoff & Sa 09 (IAN-188967); R 196 (MG-225778) & Amz, Caa, Cer, Atl, Pnt & $\mathrm{Sr}$ & $\mathrm{Vi}$ \\
\hline Hymenaea velutina Ducke & $\begin{array}{l}\text { Mo } 2760 \text { (MG-211453), Mo } 3192 \text { (MG- } \\
\text { 214821); S } 18 \text { (MG-174297); R } 54 \text { (MG- } \\
\text { 225217), R } 131 \text { (MG-225713); Sa } 12 \text { (IAN- } \\
\text { 187622); Ca 08 (MAR-6589) }\end{array}$ & Caa, Cer & Sr/Rfo & $\mathrm{Tr} / \mathrm{Sh}$ \\
\hline Indigofera microcarpa Desv. & Mo 3038 (MG-214667); R 68 (MG-225231) & Caa, Atl & $\mathrm{Sc}$ & $\mathrm{He}$ \\
\hline Leptolobium nitens Vogel + & S 64 (IAN-181087) & Amz & Rfo & $\operatorname{Tr}$ \\
\hline Mimosa sp. & $\begin{array}{c}\text { Mo } 3135 \text { (MG-214764), Mo } 3231 \text { (MG- } \\
\text { 214860); R } 185 \text { (MG-225767) }\end{array}$ & - & $\mathrm{Sr}$ & Sh \\
\hline Periandra mediterranea (Vell.) Taub. & $\begin{array}{c}\text { R } 217 \text { (MG-225799), R } 278 \text { (MG-228250); S } \\
48 \text { (MAR-6169) }\end{array}$ & Amz, Caa, Cer, Atl & Sr & $\mathrm{Sh}$ \\
\hline
\end{tabular}


Vascular flora of Lençóis Maranhenses National Park, Maranhão State, Brazil: checklist, floristic affinities and phytophysiognomies of restingas in the municipality of Barreirinhas

Table 1. Cont.

\begin{tabular}{|c|c|c|c|c|}
\hline Family/ Species & Voucher & Occurence by biome & Habitat & Habit \\
\hline Plathymenia reticulata Benth. & В 30 (MG-154866); Me 85 (MG-174784) & Amz, Caa, Cer, Atl & Rfo & $\operatorname{Tr}$ \\
\hline Poincianella pyramidalis (Tul.) L.P.Queiroz & Marc s.n.(MAR-7302) & $\mathrm{Amz}, \mathrm{Caa}$ & Rfo & $\mathrm{Tr} / \mathrm{Sh}$ \\
\hline Pterocarpus santalinoides L’Hér. ex DC. & R 280 (MG-228552) & Amz, Cer, Pnt & $\mathrm{Sr}$ & $\operatorname{Tr}$ \\
\hline Senna obtusifolia (L.) H.S. Irwin \& Barneby & R 152 (MG-225734) & Amz, Caa, Cer, Atl, Pmp, Pnt & $\mathrm{Sr}$ & $\mathrm{He} / \mathrm{Sh}$ \\
\hline $\begin{array}{l}\text { Senna pendula (Humb. \& Bonpl. ex Willd.) H.S.Irwin \& } \\
\text { Barneby }\end{array}$ & R 241 (MG-228213) & Amz, Caa, Cer, Atl, Pnt & $\mathrm{Sr}$ & $\begin{array}{l}\mathrm{Sh} / \mathrm{Tr} / \\
\mathrm{Vi}\end{array}$ \\
\hline Stryphnodendron coriaceum Benth. & $\begin{array}{l}\text { R } 199 \text { (MG-225781), R } 233 \text { (MG-225815); Sa } \\
20 \text { (MAR-5996); Mo } 3107 \text { (MG-214736) }\end{array}$ & Caa, Cer & Rfi & $\operatorname{Tr}$ \\
\hline Stylosanthes angustifolia Vogel & $\begin{array}{l}\text { Mo } 3080 \text { (MG-214709), Mo } 3138 \text { (MG- } \\
\text { 214767), Mo 3181 (MG-214810); R } 222 \\
\text { (MG-225804), R } 267 \text { (MG-228239) }\end{array}$ & Amz, Caa, Cer, Atl & Sr/Rfi & Sh \\
\hline Stylosanthes guianensis (Aubl.) Sw. & S 33 (MG-178151); Mo 3202 (MG-214831) & Amz, Caa, Cer, Atl, Pmp, Pnt & $\mathrm{Sr}$ & $\mathrm{He}$ \\
\hline Vatairea sericea (Ducke) Ducke + & B 17 (MG-154816) & $A m z$ & Rfo & $\operatorname{Tr}$ \\
\hline Zornia latifolia Sm. & $\begin{array}{c}\text { Mo } 3145 \text { (MG-214774), Mo } 3153 \text { (MG- } \\
\text { 214782); Sa } 10 \text { (IAN-188968) }\end{array}$ & Amz, Caa, Cer, Atl, Pmp, Pnt & Rfi & $\mathrm{He}$ \\
\hline \multicolumn{5}{|l|}{ GENTIANACEAE } \\
\hline Schultesia guianensis (Aubl.) Malme & $\begin{array}{c}\text { Mo } 3043 \text { (MG-214672); R } 16 \text { (MG-225179), } \\
\text { R } 78 \text { (MG-225241), R } 211 \text { (MG-225793), R } \\
277 \text { (MG-228249); Sa } 27 \text { (IAN-187633); Ca } \\
\text { 16 (IAN-185240) }\end{array}$ & Amz, Caa, Cer, Atl & $\begin{array}{l}\text { Rfi/Il/ } \\
\text { Sr }\end{array}$ & $\mathrm{He}$ \\
\hline \multicolumn{5}{|l|}{ HELIOTROPIACEAE } \\
\hline Euploca polyphylla (Lehm.) J.I.M.Melo \& Semir & $\begin{array}{c}\text { R } 74 \text { (MG-225237), R } 170 \text { (MG-225752), R } \\
194 \text { (MG-225776), R } 220 \text { (MG-225802); Mo } \\
3063 \text { (MG-214692); S } 24 \text { (MG-178601); Sa } 28 \\
\text { (MAR-5960) }\end{array}$ & Amz, Caa, Atl & Sr/Rfi & $\mathrm{He}$ \\
\hline \multicolumn{5}{|l|}{ HUMIRIACEAE } \\
\hline Humiria balsamifera (Aubl.) A.St.-Hil. & $\begin{array}{c}\text { Mo3121 (MG-214750), Mo } 3193 \text { (MG- } \\
\text { 214822); R } 32 \text { (MG-225195), R } 132 \\
\text { (MG-225714), R } 269 \text { (MG-228241); S } 18 \\
\text { (MAR-6055), S } 20 \text { (MG-174309); Ca 02 (IAN- } \\
\text { 185226) }\end{array}$ & Amz, Caa, Cer, Atl & Sr/Rfo & $\mathrm{Tr} / \mathrm{Sh}$ \\
\hline \multicolumn{5}{|l|}{ HYPERICACEAE } \\
\hline Vismia guianensis (Aubl.) Choisy & R 300 (MG-228272) & Amz, Caa, Cer, Atl & $\mathrm{Sr}$ & $\mathrm{Sh} / \mathrm{Tr}$ \\
\hline \multicolumn{5}{|l|}{ KRAMERIACEAE } \\
\hline Krameria tomentosa A.St.-Hil. & $\begin{array}{l}\text { Mo } 3104 \text { (MG-214733); R } 85 \text { (MG-225248), } \\
\text { R } 299 \text { (MG-228271); Sa s.n. (MAR-6075), Sa } \\
\text { s.n. (MAR-6076) }\end{array}$ & Amz, Caa, Cer, Atl & Sr/Rfo & Sh \\
\hline \multicolumn{5}{|l|}{ LAMIACEAE } \\
\hline Amasonia campestris (Aubl.) Moldenke & $\begin{array}{c}\text { Mo } 3212 \text { (MG-214841), Mo } 3234 \text { (214863); } \\
\text { R } 240 \text { (MG-225822) }\end{array}$ & Amz, Caa, Cer, Atl & Sr & Sh \\
\hline Hyptis atrorubens Poit. & R 189 (MG-225771) & Amz, Cer, Atl & $\mathrm{Sr}$ & $\mathrm{He}$ \\
\hline \multicolumn{5}{|l|}{ LAURACEAE } \\
\hline Cassytha filiformis L. & $\begin{array}{l}\text { Mo } 3115 \text { (MG-214744); R } 39 \text { (MG-225202), } \\
\text { R } 184 \text { (MG-225766), R } 257 \text { (MG-228229); Sa } \\
39 \text { (MAR-5965); S } 03 \text { (MG-174305) }\end{array}$ & Amz, Caa, Cer, Atl & Sr & $\mathrm{Vi}$ \\
\hline \multicolumn{5}{|l|}{ LECYTHIDACEAE } \\
\hline Eschweilera decolorans Sandwith + & В 023 (MG-154819) & $\mathrm{Amz}$ & $\mathrm{Sr}$ & $\operatorname{Tr}$ \\
\hline \multicolumn{5}{|l|}{ LENTIBULARIACEAE } \\
\hline Utricularia adpressa Salzm. ex A.St.-Hil. \& Girard. & $\begin{array}{l}\text { Mo } 3178 \text { (MG-214807), Mo } 3183 \text { (MG- } \\
\text { 214812) }\end{array}$ & Amz, Cer & Rfi & $\mathrm{He}$ \\
\hline Utricularia benjaminiana Oliv. + & R 244 (MG-228216) & $\mathrm{Amz}$ & Rfi & $\mathrm{He}$ \\
\hline Utricularia cornuta Michx. & $\begin{array}{l}\text { Mo } 3177 \text { (MG-214806), Mo } 3044 \text { (MG- } \\
\text { 214673); R } 10 \text { (MG-225173), R } 92 \text { (MG- } \\
\text { 225255), R } 246 \text { (MG-228218), R } 303 \text { (MG- } \\
\text { 228275), R } 304 \text { (MG-228276) }\end{array}$ & Cer & $\begin{array}{l}\text { Rfi/Il/ } \\
\text { Sc }\end{array}$ & $\mathrm{He}$ \\
\hline Utricularia myriocista A.St.-Hil. \& Girard. + & Mo 3125 (MG-214754) R 302 (MG-228274) & Amz, Cer, Atl & Rfi & $\mathrm{He}$ \\
\hline Utricularia simulans Pilg. & Mo 3172 (MG-214801) & Amz, Caa, Cer, Atl & Rfi & $\mathrm{He}$ \\
\hline
\end{tabular}


Misael Lira Rodrigues, Nara Furtado de Oliveira Mota, Pedro Lage Viana, Ana Kelly Koch and Ricardo de S. Secco

Table 1. Cont.

\begin{tabular}{|c|c|c|c|c|}
\hline Family/ Species & Voucher & Occurence by biome & Habitat & Habit \\
\hline Utricularia subulata $\mathrm{L}$. & $\begin{array}{c}\text { R } 8 \text { (MG-225171), R } 247 \text { (MG-228219), R } \\
248 \text { (MG-228220), R } 294 \text { (MG-228266); Mo } \\
3045 \text { (MG-214674), Mo } 3046 \text { (MG-214675), } \\
\text { Mo } 3154 \text { (MG-214783), Mo } 3189 \text { (MG- } \\
\text { 214818) }\end{array}$ & Amz, Caa, Cer, Atl & $\begin{array}{l}\text { Rfi/ll/ } \\
\text { Sr }\end{array}$ & $\mathrm{He}$ \\
\hline \multicolumn{5}{|l|}{ LORANTHACEAE } \\
\hline Passovia pedunculata (Jacq.) Kuijt & $\begin{array}{c}\text { B } 61 \text { (MG-154828), B } 66 \text { (MG-154876); Mo } \\
3182 \text { (MG-214811) }\end{array}$ & Amz, Cer & Rfi & $\mathrm{He}$ \\
\hline Passovia ovata (Pohl ex DC.) Eichler & S 10 (MG-174310) & Amz, Caa, Cer & $\mathrm{Sr}$ & $\mathrm{He}$ \\
\hline Psittacanthus robustus (Mart.) Mart. & R 02 (MG-225165) & Amz, Caa, Cer, Atl, Pnt & Sc & $\mathrm{He}$ \\
\hline \multicolumn{5}{|l|}{ LYTHRACEAE } \\
\hline Cuphea antisyphilitica Kunth. & $\begin{array}{c}\text { Mo } 3100 \text { (MG-214729), Mo } 3171 \text { (MG- } \\
\text { 214800); R } 245 \text { (MG-228217) }\end{array}$ & Amz, Cer, Atl & Sr/Rfi & Sh \\
\hline Cuphea ericoides Cham. \& Schltdl. & $\begin{array}{c}\text { R } 27 \text { (MG-225190), R } 119 \text { (MG-225701), R } \\
191 \text { (MG-225773); Mo } 3089 \text { (MG-214718); S } \\
\text { 04 (IAN-181091) }\end{array}$ & Caa, Cer & Sr/Rfi & Sh \\
\hline Lafoensia pacari A.St.-Hill. & B 13 (MG-146979) & Cer & $\mathrm{Sr}$ & $\operatorname{Tr}$ \\
\hline \multicolumn{5}{|l|}{ MALPIGHIACEAE } \\
\hline Byrsonima laevis Nied. + & $\begin{array}{l}\text { R } 130 \text { (MG-225712), R } 232 \text { (MG-225814); } \\
\text { Mo } 3110 \text { (MG-214739); B } 14 \text { (MG-154813) }\end{array}$ & $\operatorname{Amz}$ & $\mathrm{Sc} / \mathrm{Sr}$ & $\operatorname{Tr}$ \\
\hline Byrsonima sericea DC. & $\begin{array}{l}\text { R } 04 \text { (MG-225167), R } 129 \text { (MG-225711), R } \\
275 \text { (MG-228247); S } 13 \text { (MG-174301) }\end{array}$ & Amz, Caa, Cer, Atl & $\mathrm{Sc} / \mathrm{Sr}$ & Sh \\
\hline Byrsonima sp. & Mo 3128 (MG-214757) S 09 (MG-174302) & - & Sr & $\mathrm{Tr} / \mathrm{Sh}$ \\
\hline Heteropterys nervosa A.Juss. + & S 05 (MG-174312); Ca 03 (MAR-6591) & Amz, Cer, Atl & $\mathrm{Sc} / \mathrm{Sr}$ & Vi \\
\hline Niedenzuella sp. & В 02 (MG-146969) & - & - & $\mathrm{Vi}$ \\
\hline Stigmaphyllon paralias A.Juss. & $\begin{array}{c}\text { S } 34 \text { (MG-178152); R } 125 \text { (MG-225707), R } \\
159 \text { (MG-225741); Mo } 3074 \text { (MG-214703); B } \\
34 \text { (MG-154867) }\end{array}$ & Caa, Cer, Atl & $\mathrm{Sr}$ & Sh \\
\hline \multicolumn{5}{|l|}{ MALVACEAE } \\
\hline Sida castanocarpa Kaprov. & Sa 13 (IAN-188970) & Caa, Cer & $\mathrm{Sr}$ & $\mathrm{He}$ \\
\hline Sida cordifolia L. & R 067 (MG-225230) & Amz, Caa, Cer, Atl & Sc & $\mathrm{He}$ \\
\hline Sterculia sp. & Sa 11 (IAN-187621) & - & - & Sh \\
\hline \multicolumn{5}{|l|}{ MAYACACEAE } \\
\hline Mayaca longipes Mart. ex Seub. + & Mo 3224 (MG-214853) & Amz, Cer, Atl, Pnt & Rfi/Il & $\mathrm{He}$ \\
\hline \multicolumn{5}{|l|}{ MELASTOMATACEAE } \\
\hline Comolia villosa (Aubl.) Triana & $\begin{array}{c}\text { S } 35 \text { (MG-178153); Mo } 3220 \text { (MG- } \\
\text { 214849), Mo } 3023 \text { (MG-214652, Mo } 3097 \\
\text { (MG-214726); R } 47 \text { (MG-225210), R } 115 \\
\text { (MG-225697), R } 190 \text { (MG-225772), R } 219 \\
\text { (MG-225801), R } 263 \text { (MG-228235), R } 282 \\
\text { (MG-228254), R } 305 \text { (MG-228277); Ca } 11 \\
\text { (IAN-185235) }\end{array}$ & Amazônica, Caa, Atl & Sr/Rfi & Sh \\
\hline Mouriri guianensis Aubl. & $\begin{array}{l}\text { R } 35 \text { (MG-225198), R } 134 \text { (MG-225716), R } \\
235 \text { (MG-225817); Mo } 3129 \text { (MG-214758); } \\
\text { Ca } 11 \text { (MAR-6585), Ca } 17 \text { (IAN-185241); B } \\
\text { 26 (MG-154821) }\end{array}$ & Amz, Cer, Atl, Pnt & $\mathrm{Sr} / \mathrm{Sc}$ & $\mathrm{Sh} / \mathrm{Tr}$ \\
\hline Siphanthera cordifolia (Benth.) Gleason + & $\begin{array}{c}\text { Sa } 36 \text { (MAR-5992); R } 11 \text { (MG-225174), R } 14 \\
\text { (MG-225177), R } 251 \text { (MG-228223) }\end{array}$ & Amz, Atl & Rfi/Il & $\mathrm{He}$ \\
\hline \multicolumn{5}{|l|}{ MENYANTHACEAE } \\
\hline Nymphoides humboldtiana (Kunth) Kuntze & $\begin{array}{l}\text { Mo } 3042 \text { (MG-214671); R } 187 \text { (MG-225769), } \\
\text { R } 301 \text { (MG-228273); Sa } 31 \text { (MAR-5995) }\end{array}$ & Amz, Caa, Cer, Atl, Pmp, Pnt & Rfi/Il & $\mathrm{He}$ \\
\hline \multicolumn{5}{|l|}{ MYRTACEAE } \\
\hline Eugenia biflora (L.) DC. & R 44 (MG-225207), R 287 (MG-228259) & Amz, Caa, Cer & $\mathrm{Sr}$ & $\mathrm{Sh} / \operatorname{Tr}$ \\
\hline Eugenia punicifolia (Kunth.) DC. & Mo 3207 (MG-214836) & Amz, Caa, Cer, Atl & $\mathrm{Sr}$ & Sh \\
\hline Eugenia sp. & $\begin{array}{l}\text { R } 162 \text { (MG-225744), R } 164 \text { (MG-225746); B } \\
38 \text { (MG-154870); Mo } 3214 \text { (MG-214843); S } \\
\text { 30 (MG-173148) }\end{array}$ & - & $\mathrm{Sr}$ & Sh \\
\hline
\end{tabular}


Vascular flora of Lençóis Maranhenses National Park, Maranhão State, Brazil: checklist, floristic affinities and phytophysiognomies of restingas in the municipality of Barreirinhas

Table 1. Cont.

\begin{tabular}{|c|c|c|c|c|}
\hline Family/Species & Voucher & Occurence by biome & Habitat & Habit \\
\hline Myrcia multiflora (Lam.) DC. & $\begin{array}{c}\text { R } 22 \text { (MG225185), R } 36 \text { (MG-225199), R } 182 \\
\text { (MG-225764), R } 268 \text { (MG-228240) }\end{array}$ & Amz, Caa, Cer, Atl & Rfo & $\mathrm{Sh} / \mathrm{Tr}$ \\
\hline Myrcia splendens (Sw.) DC. & $\begin{array}{l}\text { R } 41 \text { (MG-225204), R } 156 \text { (MG-225738); B } \\
65 \text { (MG-154830) }\end{array}$ & Amz, Caa, Cer, Atl & Rfo & $\operatorname{Tr}$ \\
\hline Myrcia sylvatica (G.Mey) DC. & Mo 3070 (MG-214699) & $\mathrm{Amz}, \mathrm{Caa}, \mathrm{Cer}$ & Rfo & $\operatorname{Tr}$ \\
\hline Myrcia tomentosa (Aubl.) DC. & R 59 (MG-225222); B 70 (MG-154830) & Amz, Caa, Cer, Atl & $\mathrm{Sr}$ & $\operatorname{Tr}$ \\
\hline Myrcia sp. & R 38 (MG-225201), R 43 (MG-225206) & - & Rfo & $\operatorname{Tr}$ \\
\hline Myrciaria floribunda (H.West ex Willd.) O.Berg. + & B 25 (MG-154820) & Amz, Caa, Cer, Atl & Rfo & $\operatorname{Tr}$ \\
\hline \multicolumn{5}{|l|}{ NYCTAGYNACEAE } \\
\hline Guapira pernambucensis (Casar.) Lundell. & Mo 3161 (MG-214790); Sa s.n. (MAR-6628) & Atl & $\mathrm{Sr}$ & Sh \\
\hline \multicolumn{5}{|l|}{ OCHNACEAE } \\
\hline Ouratea caudata Engl. & $\begin{array}{l}\text { Mo } 3108 \text { (MG-214737); R } 34 \text { (MG-225197), } \\
\text { R } 158 \text { (MG-225740), R } 175 \text { (MG-225757), R } \\
285 \text { (MG-228257), R } 298 \text { (MG-228270); Ca } \\
06 \text { (IAN-185220), Ca } 20 \text { (IAN-185244); S } 15 \\
\text { (MG-174313) }\end{array}$ & $\mathrm{Amz}, \mathrm{Cer}$ & Sr & Sh \\
\hline Sauvagesia sp. & Mo 3176 (MG-214805) & - & $\mathrm{Sr}$ & $\mathrm{Sh} / \mathrm{He}$ \\
\hline \multicolumn{5}{|l|}{ ONAGRACEAE } \\
\hline Ludwigia leptocarpa (Nutt.) H.Hara & Cb 15 (MAR-2646) & Amz, Caa, Cer, Atl, Pnt & Rfi & $\mathrm{Sh} / \mathrm{E}$ \\
\hline Ludwigia octovalvis (Jacq.) P.H.Raven + & Mo 3230 (MG-214859) & Amz, Caa, Cer, Atl, Pnt & Rfi & $\mathrm{Sh} / \mathrm{He}$ \\
\hline Ludwigia sp. & $\begin{array}{c}\text { Mo } 3190 \text { (MG-214819); R } 75 \text { (MG-225238); } \\
\text { Ex s.n. (MAR-3244) }\end{array}$ & - & Rfi & $\mathrm{He}$ \\
\hline \multicolumn{5}{|l|}{ ORCHIDACEAE } \\
\hline Habenaria depressifolia Hoehne + & Mo 3201 (MG-214830) & $\mathrm{Amz}, \mathrm{Cer}$ & Rfi & $\mathrm{He}$ \\
\hline Habenaria leprieuri Rchb.F. + & Mo 3175 (MG-214804) & Amz, Cer & Rfi & $\mathrm{He}$ \\
\hline Habenaria ludibundiciliata J.A.N.Batista \& Bianch & Mo 3198 (MG-214827) & $\mathrm{Amz}, \mathrm{Cer}$ & Rfi & $\mathrm{He}$ \\
\hline Habenaria orchiocalcar Hoehne & Mo 3173 (MG-214802) & Amz, Cer & Rfi & $\mathrm{He}$ \\
\hline Habenaria sprucei Cogn. & $\begin{array}{c}\text { Mo } 3165 \text { (MG-214794), Mo } 3079 \text { (MG- } \\
\text { 214708) }\end{array}$ & Amz, Caa, Cer & Rfi & $\mathrm{He}$ \\
\hline $\begin{array}{c}\text { Trichocentrum sprucei (Lindl.) M.W.Chase \& } \\
\text { N.H.Williams + }\end{array}$ & R 283 (MG-228255) & $\mathrm{Amz}$ & Rfo & $\mathrm{He}$ \\
\hline \multicolumn{5}{|l|}{ PASSIFLORACEAE } \\
\hline Passiflora foetida L. & Mo 3191 (MG-214820); R 64 (MG-225227) & Amz, Caa, Cer, Atl, Pmp, Pnt & $\mathrm{Sr}$ & $\mathrm{Vi}$ \\
\hline \multicolumn{5}{|l|}{ PENTAPHYLACACEAE } \\
\hline Ternstroemia delicatula Choisy + & Ca 10 (MAR-6593) & $\mathrm{Amz}$ & $\mathrm{Sr}$ & $\mathrm{Sh}$ \\
\hline \multicolumn{5}{|l|}{ PHYLLANTACEAE } \\
\hline Phyllanthus hyssopifolioides Kunth + & Mo 3227 (MG-214856) & $\mathrm{Amz}, \mathrm{Cer}$ & $\mathrm{Sr}$ & $\mathrm{He}$ \\
\hline Phyllanthus orbiculatus Rich. & Mo 3209 (MG-214838) & Amz, Caa, Cer & Sr & $\mathrm{He}$ \\
\hline Phyllanthus stipulatus (Raf.) G.L.Webster & Mo 3065 (MG-214694) & Amz, Cer, Atl & $\mathrm{Sr}$ & $\mathrm{He}$ \\
\hline \multicolumn{5}{|l|}{ PLANTAGINACEAE } \\
\hline Bacopa cochlearia (Huber) L.B.Sm. ${ }_{+}+$ & R 15 (MG-225178) & Caa & Il & $\mathrm{He}$ \\
\hline Bacopa imbricata (Benth.) Pennell & R 319 (MG-228291) & $\mathrm{Amz}$ & $\mathrm{Sc}$ & $\mathrm{He}$ \\
\hline Bacopa reflexa (Benth.) Edwall & Mo 3047 (MG-214676) & Amz, Cer & Rfi & $\mathrm{He}$ \\
\hline Bacopa salzmannii (Benth.) Wettst. ex Edwall & Mo 3037 (MG-214666) & Amz, Caa, Cer, Atl, Pnt & $\mathrm{Sc}$ & $\mathrm{He}$ \\
\hline Dizygostemon sp. & Mo 3099 (MG-214728) & Caa & Rfi & $\mathrm{He}$ \\
\hline Tetraulacium veroniciforme Turcz. & $\begin{array}{c}\text { Mo } 3067 \text { (MG-214696), Mo } 3131 \text { (MG- } \\
\text { 214760) }\end{array}$ & Caa, Atl, Pnt & Rfi & $\mathrm{He}$ \\
\hline \multicolumn{5}{|l|}{ POACEAE } \\
\hline Andropogon bicornis $\mathrm{L}$ & R 176 (MG-225758) & Amz, Caa, Cer, Atl, Pmp, Pnt & Sr & $\mathrm{He}$ \\
\hline Andropogon leucostachyus Kunth. & $\begin{array}{l}\text { R } 24 \text { (MG-225187), R } 177 \text { (MG-225759), R } \\
206 \text { (MG-225788); Mo } 3028 \text { (MG-214657) }\end{array}$ & Amz, Caa, Cer, Atl, Pmp, Pnt & $\mathrm{Sr} / \mathrm{Sc}$ & $\mathrm{He}$ \\
\hline Andropogon virgatus Desv. & Mo 3088 (MG-214717) & Amz, Caa, Cer, Atl, Pmp & Rfi & $\mathrm{He}$ \\
\hline Aristida longifolia Trin. & $\begin{array}{c}\text { Mo } 3105 \text { (MG-214734); R } 80 \text { (MG-225243), } \\
\text { R } 288 \text { (MG-228260) }\end{array}$ & $\mathrm{Amz}, \mathrm{Caa}, \mathrm{Cer}$ & Sr/Rfi & $\mathrm{He}$ \\
\hline Axonopus capillaris (Lam.) Chase & $\begin{array}{c}\text { Mo } 3056 \text { (MG-214685), Mo } 3215 \text { (MG- } \\
\text { 214844) }\end{array}$ & Amz, Caa, Cer, Atl & Sr/Rfi & $\mathrm{He}$ \\
\hline
\end{tabular}




\section{Misael Lira Rodrigues, Nara Furtado de Oliveira Mota, Pedro Lage Viana, Ana Kelly Koch and Ricardo de S. Secco}

Table 1. Cont.

\begin{tabular}{|c|c|c|c|c|}
\hline Family/ Species & Voucher & Occurence by biome & Habitat & Habit \\
\hline Axonopus polydactylus (Steud.) Dedecca & $\begin{array}{c}\text { Mo } 3058 \text { (MG-214687), Mo } 3141 \text { (MG- } \\
\text { 214770); R } 179 \text { (MG-225761) }\end{array}$ & Cer, Atl & Sr/Rfi & $\mathrm{He}$ \\
\hline Axonopus purpusii (Mez.) Chase & $\begin{array}{c}\text { Mo } 3075 \text { (MG-214704), Mo } 3137 \text { (MG- } \\
\text { 214766); R } 29 \text { (MG-225192) }\end{array}$ & Amz, Caa, Cer, Atl & Sr/Rfi & $\mathrm{He}$ \\
\hline Cenchrus brownii Roem. \& Schult. + & $\begin{array}{l}\text { Mo } 3142 \text { (MG-214771); } \\
\text { R } 70 \text { (MG-225233) }\end{array}$ & Amz, Caa, Cer, Atl, Pnt & $\mathrm{Cr} / \mathrm{Sc}$ & $\mathrm{He}$ \\
\hline Coleataenia stenodes (Griseb) Soreng. + & Mo 3085 (MG-214714) & $\mathrm{Amz}, \mathrm{Cer}$ & Rfi & $\mathrm{He}$ \\
\hline $\begin{array}{l}\text { Cyphonathus discrepans (Döll) } \\
\text { Zuloaga \& Morrone }\end{array}$ & Mo 3050 (MG-214679) & Amz, Cer, Atl & Rfi & $\mathrm{He}$ \\
\hline Digitaria cuyabensis (Trin.) Parodi + & $\begin{array}{l}\text { Mo } 3158 \text { (MG-214787); } \\
\text { R } 213 \text { (MG-225795) }\end{array}$ & Caa, Cer, Atl, Pmp, Pnt & Sc & $\mathrm{He}$ \\
\hline Eragrostis guianensis Hitchc. + & $\begin{array}{l}\text { R } 83 \text { (MG-225246), R } 202 \text { (MG-225784), R } \\
229 \text { (MG-225811), R } 230 \text { (MG-225812); Mo } \\
\text { 3027 (MG-214656) }\end{array}$ & $\mathrm{Amz}$ & Sc/Rfi & $\mathrm{He}$ \\
\hline Eragrostis maypurensis (Kunth) Steud & R 289 (MG-228261) & Amz, Caa, Cer, Atl & $\mathrm{Sr}$ & $\mathrm{He}$ \\
\hline Gymnopogon foliosus (Willd.) Nees & R 48 (MG-225211) & Amz, Caa, Cer, Atl & $\mathrm{Sr}$ & $\mathrm{He}$ \\
\hline Homolepis isocalycia (G. Mey.) Chase & S 44 (MAR-6061) & Amz, Caa, Cer, Atl & $\mathrm{Sr}$ & $\mathrm{He}$ \\
\hline Mesosetum loliiforme (Hochst. ex Steud.) Chase & Mo 3052 (MG-214681) & Amz, Caa, Cer, Atl, Pnt & Sr/Rfi & $\mathrm{He}$ \\
\hline Mesosetum sp. nov. & $\begin{array}{l}\text { R 72, 93, R } 205 \text { (MG-225787), 210; } \\
\text { Mo 3040, } 3112\end{array}$ & - & $\mathrm{Rfi} / \mathrm{Sc}$ & $\mathrm{He}$ \\
\hline Panicum aquaticum Poir. & Mo 3041 (MG-214670) & Amz, Caa, Cer, Atl, Pmp, Pnt & Sc/Il & $\mathrm{He}$ \\
\hline Panicum trichoides Sw. & Mo 3208 (MG-214837) & Amz, Caa, Cer, Atl, Pnt & $\mathrm{Sr}$ & $\mathrm{He}$ \\
\hline Paspalidium geminatum (Forssk.) Stapf. & Mo 3021 (MG-214650) & Amz, Caa, Atl, Pnt & Sc & $\mathrm{He}$ \\
\hline Paspalum carinatum Humb. \& Bonpl. ex Flüggé & Mo 3076 (MG-214705) & Amz, Caa, Cer, Atl, Pnt & Sr/Rfi & $\mathrm{He}$ \\
\hline Paspalum ligulare Nees & Mo 3033 (MG-214662) & Amz, Caa, Atl & Sc & $\mathrm{He}$ \\
\hline Paspalum pulchellum Kunth & $\begin{array}{c}\text { Mo } 3170 \text { (MG-214799), Mo } 3077 \text { (MG- } \\
\text { 214706); R } 28 \text { (MG-225191) }\end{array}$ & Amz, Cer & Sr/Rfi & $\mathrm{He}$ \\
\hline Paspalum pumilum Nees + & Mo 3062 (MG-214691) & Amz, Cer, Atl, Pmp & $\mathrm{Sr}$ & $\mathrm{He}$ \\
\hline Paspalum spissum Swallen & R 178 (MG-225760) & $\mathrm{Amz}, \mathrm{Cer}$ & $\mathrm{Sr}$ & $\mathrm{He}$ \\
\hline Paspalum vaginatum $\mathrm{Sw}$. & R 225 (MG-225807); Mo 3031 (MG-214660) & Amz, Caa, Cer, Atl, Pmp, Pnt & $\mathrm{Hv}$ & $\mathrm{He}$ \\
\hline Reimarochloa aberrans (Döll) Chase + & R 214 (MG-225796); Mo 3159 (MG-214788) & $\mathrm{Amz}$ & Sc & $\mathrm{He}$ \\
\hline Rhytachne guianensis (Hitchc.) Clayton + & Mo 3186 (MG-214815) & $\mathrm{Amz}$ & Rfi & $\mathrm{He}$ \\
\hline Sacciolepis vilvoides (Trin.) Chase + & R 256 (MG-228228) & Amz, Caa, Cer, Atl & $\mathrm{Sr}$ & $\mathrm{He}$ \\
\hline Spartina alterniflora Loisel. & $\begin{array}{c}\text { R } 89 \text { (MG-225252), R } 223 \text { (MG-225805), } \\
\text { R } 313 \text { (MG-228285) }\end{array}$ & Amz, Atl, Pmp & $\mathrm{Hv} / \mathrm{Sc}$ & $\mathrm{He}$ \\
\hline Sporobolus virginicus (L.) Kunth & $\begin{array}{l}\text { R } 87 \text { (MG-225250), R } 226 \text { (MG-225808); } \\
\text { Mo } 3034 \text { (MG-214663) }\end{array}$ & Amz, Caa, Cer, Atl, Pmp & $\mathrm{Hv}$ & $\mathrm{He}$ \\
\hline Streptostachys asperifolia Desv. & $\begin{array}{l}\text { R } 166 \text { (MG-225748); Mo } 3054 \text { (MG-214683), } \\
\text { Mo } 3211 \text { (MG-214840) }\end{array}$ & Amz, Caa, Cer, Atl & $\mathrm{Sr}$ & $\mathrm{He}$ \\
\hline Trachypogon spicatus (L.f.) Kuntze & $\begin{array}{c}\text { R } 06 \text { (MG-225169), R } 07 \text { (MG-225170), R } \\
150 \text { (MG-225732), R } 203 \text { (MG-225785) R } \\
207 \text { (MG225789), R } 307 \text { (MG-228279); Mo } \\
3078 \text { (MG-214707), Mo } 3111 \text { (MG-214740) }\end{array}$ & Amz, Caa, Cer, Atl & Rfi & $\mathrm{He}$ \\
\hline Trachypogon vestitus Andersson & Sa 02 (MAR-6095) & Amz, Caa, Cer, Atl & $\mathrm{Sr}$ & $\mathrm{He}$ \\
\hline $\begin{array}{l}\text { Trichanthecium polycomum (Trin.) } \\
\text { Zuloaga \& Morrone + }\end{array}$ & Mo 2775 (MG-211468); R 46 (MG-225209) & Amz, Caa, Atl & $\mathrm{Sr}$ & $\mathrm{He}$ \\
\hline $\begin{array}{l}\text { Trichanthecium nervosum (Lam.) } \\
\text { Zuloaga \& Morrone + }\end{array}$ & Mo 3084 (MG-214713) & Amz, Caa, Cer, Atl, Pnt & Rfi & $\mathrm{He}$ \\
\hline \multicolumn{5}{|l|}{ POLYGALACEAE } \\
\hline $\begin{array}{c}\text { Asemeia rhodoptera (Mart. ex A.W.Benn.) } \\
\text { J.F.B.Pastore \& J.R. Abbott + }\end{array}$ & Mo 3096 (MG-214725) & Cer & Rfi & $\mathrm{He}$ \\
\hline Polygala adenophora DC. & Mo 3222 (MG-214851) & Amz, Cer, Atl & Sr/Rfi & $\mathrm{He}$ \\
\hline Polygala appressa Benth. & $\begin{array}{c}\text { Mo } 3083 \text { (MG-214712), Mo } 3236 \text { (MG- } \\
\text { 214865); R } 117 \text { (MG-225699) }\end{array}$ & Amz, Cer, Atl & Sr/Rfi & $\mathrm{He}$ \\
\hline Polygala glochidata Kunth & $\begin{array}{l}\text { Mo } 3039 \text { (MG-214668), Mo } 3143 \text { (MG- } \\
\text { 214772) }\end{array}$ & Amz, Caa, Cer, Atl & Rfi & $\mathrm{He}$ \\
\hline
\end{tabular}


Vascular flora of Lençóis Maranhenses National Park, Maranhão State, Brazil: checklist, floristic affinities and phytophysiognomies of restingas in the municipality of Barreirinhas

Table 1. Cont.

\begin{tabular}{|c|c|c|c|c|}
\hline Family/Species & Voucher & Occurence by biome & Habitat & Habit \\
\hline Polygala sedoides A.W.Benn & R 306 (MG-228278) & Amz, Cer, Atl & Rfi & $\mathrm{He}$ \\
\hline Polygala subtilis Kunth & Mo 3095 (MG-214724) & Amz, Cer & Rfi & $\mathrm{He}$ \\
\hline Polygala trichosperma Jacq. & $\begin{array}{c}\text { Mo } 3098 \text { (MG-214727), Mo 3134 (MG- } \\
\text { 214763), Mo } 3229 \text { (MG-214858); R } 25 \\
\text { (MG-225188), R } 118 \text { (MG-225700), R } 121 \\
\text { (MG-225703), R } 123 \text { (MG-225705), R } 188 \\
\text { (MG-225770), R } 212 \text { (MG-225794), R } 292 \\
\text { (MG-228264); Ca } 01 \text { (IAN-185225), Ca } 21 \\
\text { (MAR-6598) }\end{array}$ & Amz, Caa, Cer, Atl & Sr/Rfi & $\mathrm{He}$ \\
\hline Securidaca bialata Benth & Ca 09 (IAN-185233) & Amz, Cer & $\mathrm{Sr}$ & $\mathrm{Vi}$ \\
\hline \multicolumn{5}{|l|}{ POLYGONACEAE } \\
\hline Coccoloba ramosissima Wedd. & S 29 (MG-178147) & Amz, Atl & $\begin{array}{l}\mathrm{Sr} / \mathrm{Scl} \\
\mathrm{Rfo}\end{array}$ & $\mathrm{Sh} / \mathrm{Tr}$ \\
\hline \multicolumn{5}{|l|}{ PORTULACACEAE } \\
\hline Portulaca oleracea L. & Rh 302 (MAR-1634) & Amz, Caa, Cer, Atl & $\mathrm{Es} / \mathrm{Sr}$ & $\mathrm{He}$ \\
\hline \multicolumn{5}{|l|}{ RHIZOPHORACEAE } \\
\hline Rhizophora harrisonii Leechm. & Marc s.n. (MAR-2179) & $\mathrm{Amz}$ & Gf & $\operatorname{Tr}$ \\
\hline Rhizophora racemosa G.Mey & Marc s.n. (MAR-2177) & $\mathrm{Amz}$ & Gf & $\operatorname{Tr}$ \\
\hline \multicolumn{5}{|l|}{ RUBIACEAE } \\
\hline Borreria paraensis E.L.Cabral \& Bacigalupo + & Mo 3160 (MG-214789) & Amz & $\mathrm{Sr}$ & $\mathrm{Sh} / \mathrm{He}$ \\
\hline Borreria remota (Lam.) Bacigalupo \& E.L.Cabral + & $\begin{array}{c}\text { Mo } 3057 \text { (MG-214686), Mo } 3064 \text { (MG- } \\
\text { 214693), Mo } 3118 \text { (MG-214747) }\end{array}$ & Amz, Cer, Atl & Sr & $\mathrm{Sh} / \mathrm{He}$ \\
\hline Borreria verticillata (L.) G.F.W.Mey & $\begin{array}{l}\text { O } 35 \text { (MG-133375); Mo } 3116 \text { (MG-214745), } \\
\text { Mo } 3195 \text { (MG-214824); R } 69 \text { (MG-225232), } \\
\text { R } 73 \text { (MG-225236), R } 209 \text { (MG-225791), R } \\
297 \text { (MG-228269), R } 312 \text { (MG-228284); Ca } \\
15 \text { (MAR-6342);.Sa } 03 \text { (IAN-188960) }\end{array}$ & Amz, Caa, Cer, Atl & Sr/Rfi & Sh \\
\hline Chiococca alba (L.) Hitch. & B s.n. (MG-154862) & Amz, Caa, Cer, Atl, Pnt & Sr & Sh \\
\hline Duroia paraensis Ducke + & R 284 (MG-228256) & Amamzônia & Sr & $\operatorname{Tr}$ \\
\hline Guettarda platypoda DC. & R 136 (MG-225718);.Sa 17 (MAR-6080) & Amamzônia, Atl & Sr/Rfi & Sh \\
\hline Hexasepalum apiculatum (Willd.) Delprete \& J.Kirkbr. & Sa s.n. (MAR-6608) & Amz, Caa, Cer, Pmp & $\mathrm{Sr}$ & Sh \\
\hline Hexasepalum teres (Walter) J.H.Kirkbr. & Mo 3139 (MG-214768) & Amz, Caa, Cer, Atl, Pmp, Pnt & Rfi & $\mathrm{He}$ \\
\hline $\begin{array}{c}\text { Mitracarpus strigosus (Thunb.) P.L.R.Moraes, Smedt \& } \\
\text { Hjertson }\end{array}$ & $\begin{array}{c}\text { Mo } 3066 \text { (MG-214695), Mo } 3132 \text { (MG- } \\
\text { 214761), Mo } 3147 \text { (MG-214776); R } 65 \\
\text { (MG-225228) }\end{array}$ & Amz, Caa, Cer, Atl & Sr/Rfi & $\mathrm{He}$ \\
\hline Oldenlandia tenuis K. Schum. & Mo 3146 (MG-214775) & $\mathrm{Amz}, \mathrm{Caa}$ & Rfi & $\mathrm{He}$ \\
\hline Pagamea guianensis Aubl. & $\begin{array}{c}\text { R } 37 \text { (MG-225200), R } 264 \text { (MG-228236); Mo } \\
3196 \text { (MG-214825) }\end{array}$ & Amz, Caa, Cer, Atl & Sr & $\mathrm{Sh} / \mathrm{Tr}$ \\
\hline Perama hirsuta Aubl. & Mo 3094 (MG-214723); R 128 (MG-225710) & Amz, Caa, Cer, Atl & Rfi & $\mathrm{He}$ \\
\hline $\begin{array}{l}\text { Psychotria hoffmannseggiana } \\
\text { (Willd. ex Schult) Müll.Arg. }\end{array}$ & Mo 3225 (214854); S 55 (IAN-181348) & Amz, Cer, Atl & Sr & Sh \\
\hline Staelia virgata (Link ex Roem. \& Schult.) K.Schum. & Mo 3164 (MG-214793) & Amz, Caa, Cer, Atl, Pnt & Sr & $\mathrm{Sh} / \mathrm{He}$ \\
\hline Spermacoce prostrata Aubl. + & Mo 3150 (MG-214854) & Amz, Caa, Cer, Atl & Rfi & $\mathrm{He}$ \\
\hline Tocoyena brasiliensis Mart. & Mt 31 (MAR-7093); S 43 (MAR-6059) & Amz, Caa, Cer, Atl & $\mathrm{Sr} / \mathrm{Sc}$ & $\mathrm{Sh} / \mathrm{Tr}$ \\
\hline Tocoyena hispidula Standl. & R 169 (MG-225751), R 200 (MG-225782) & $\mathrm{Amz}, \mathrm{Caa}, \mathrm{Cer}$ & Sr/Rfi & Sh \\
\hline \multicolumn{5}{|l|}{ SAPINDACEAE } \\
\hline Matayba discolor (Spreng.) Radk. + & $\begin{array}{l}\text { S } 28 \text { (MG-178603); Mo } 3061 \text { (MG-214690); R } \\
\quad 151 \text { (MG-225733), R } 181 \text { (MG-225763) }\end{array}$ & Atl & Rfo & $\mathrm{Tr} / \mathrm{Sh}$ \\
\hline \multicolumn{5}{|l|}{ SAPOTACEAE } \\
\hline Manilkara triflora (Allemão) Monach. & $\begin{array}{c}\text { Mo } 3117 \text { (MG-214776); Sa } 14 \text { (IAN-188971), } \\
\text { Sa } 35 \text { (MAR-5963) }\end{array}$ & Amz, Caa, Cer, Atl & Rfo & $\mathrm{Tr} / \mathrm{Sh}$ \\
\hline Pouteria ramiflora (Mart.) Radlk. & S 16 (MAR-6068) & Amz, Caa, Cer, Atl & Sr & $\mathrm{Sh} / \mathrm{Tr}$ \\
\hline \multicolumn{5}{|l|}{ SIMAROUBACEAE } \\
\hline $\begin{array}{l}\text { Homalolepis trichilioides (A.St.-Hil.) } \\
\text { Devecchi \& Pirani }\end{array}$ & $\begin{array}{l}\text { R } 114 \text { (MG-225257), R } 286 \text { (MG-228258); Ca } \\
07 \text { (MAR-6601); S } 19 \text { (MAR-6119) }\end{array}$ & Cer, Atl & Sr & $\operatorname{Tr}$ \\
\hline \multicolumn{5}{|l|}{ SMILACACEAE } \\
\hline Smilax syphilitica Humb. \& Bonpl. ex Willd. & R 271 (MG-228243) & Amz, Caa, Cer, Atl & Sr & $\mathrm{Vi}$ \\
\hline
\end{tabular}




\section{Misael Lira Rodrigues, Nara Furtado de Oliveira Mota, Pedro Lage Viana, Ana Kelly Koch and Ricardo de S. Secco}

Table 1. Cont.

\begin{tabular}{|c|c|c|c|c|}
\hline Family/ Species & Voucher & Occurence by biome & Habitat & Habit \\
\hline \multicolumn{5}{|l|}{ SOLANACEAE } \\
\hline Schwenckia americana Rooyen ex L. & Mo 3157 (MG- 214786) & Amz, Caa, Cer, Atl & Sr/Rfi & $\mathrm{He}$ \\
\hline Solanum paludosum Moric. & Sa s.n. (MAR-6616) & Amz, Caa, Atl & Sc & Sh \\
\hline \multicolumn{5}{|l|}{ TURNERACEAE } \\
\hline Piriqueta duarteana (Cambess) Urb. & Mo 3235 (MG-214864) & Amz, Caa, Cer, Atl & $\mathrm{Sr}$ & $\mathrm{He} / \mathrm{Sh}$ \\
\hline Turnera melochioides cf. var. arenaria Urb. & Ca 13 (MAR-6587) & $\mathrm{Amz}, \mathrm{Caa}, \mathrm{Cer}$ & $\mathrm{Sr} / \mathrm{Sc}$ & Sh \\
\hline Turnera melochioides var. latifolia Urb. & $\begin{array}{l}\text { Mo } 3200 \text { (MG-214829); R } 126 \text { (MG-225708), } \\
\quad \text { R } 153 \text { (MG-225735), R } 215 \text { (MG-225797) }\end{array}$ & Amz, Caa, Cer, Atl & $\mathrm{Sr}$ & $\mathrm{Sh}$ \\
\hline Turnera melochioides var. melochioides Cambess. & S 37 (MG-178155) & Amz, Caa, Cer, Atl & $\mathrm{Sr}$ & Sh \\
\hline \multicolumn{5}{|l|}{ VERBENACEAE } \\
\hline Casselia integrifolia Nees \& Mart. + & $\begin{array}{c}\text { S } 26 \text { (MG-178144); R } 161 \text { (MG-225743), R } \\
167 \text { (MG-225749) }\end{array}$ & Caa, Cer, Atl & $\mathrm{Sr}$ & $\mathrm{He}$ \\
\hline Lantana cf. fucata Lindl. & S 27 (MG-178145) & Caa, Cer, Atl & $\mathrm{Sr}$ & Sh \\
\hline Stachytarpheta jamaicensis (L.) Vahl & Sa 06 (MAR-188963) & Amz, Atl & $\mathrm{Sr}$ & $\mathrm{He} / \mathrm{Sh}$ \\
\hline \multicolumn{5}{|l|}{ VIOLACEAE } \\
\hline Pombalia calceolaria (L.) Paula-Souza & Mo 3152 (MG-214781); R 71 (MG-225234) & Amz, Caa, Cer, Atl, Pnt & Rfi & $\mathrm{He}$ \\
\hline \multicolumn{5}{|l|}{ VOCHYSIACEAE } \\
\hline Salvertia convallariodora A.St.-Hil. & B 15 (MG-154814) & Amz, Caa, Cer, Atl & $\mathrm{Sr}$ & $\operatorname{Tr}$ \\
\hline Qualea parviflora Mart. & S 40 (MAR-6070) & Amz, Caa, Cer, Atl & $\mathrm{Sr}$ & $\mathrm{Sh} / \mathrm{Tr}$ \\
\hline \multicolumn{5}{|l|}{ XYRIDACEAE } \\
\hline Abolboda poarchon Seub. & $\begin{array}{c}\text { Mo } 3167 \text { (MG-214796); R } 140 \text { (MG-225722), } \\
\text { R } 258 \text { (MG-228230) }\end{array}$ & $\mathrm{Amz}, \mathrm{Cer}$ & Rfi & $\mathrm{He}$ \\
\hline Xyris anceps Lam. & Cb 04 (MAR-2650) & Amz, Cer, Atl & Sr/Rfi & $\mathrm{He}$ \\
\hline Xyris fallax Malme & Mo 3168 (MG-214797) & $\mathrm{Amz}, \mathrm{Caa}, \mathrm{Cer}$ & Rfi & $\mathrm{He}$ \\
\hline Xyris jupicai Rich & $\begin{array}{c}\text { Mo } 2774 \text { (MG-211467), Mo } 3119 \text { (MG- } \\
\text { 214748); R } 19 \text { (MG-225182) }\end{array}$ & $\begin{array}{c}\text { Amz, Caa, Cer, Mata Atl, Pmp, } \\
\text { Pnt }\end{array}$ & Rfi/Il & $\mathrm{He}$ \\
\hline Xyris paraensis Poepp. ex Kunth & $\begin{array}{c}\text { Mo } 2812 \text { (MG-211505), Mo } 3188 \text { (MG- } \\
\text { 214817); R } 51 \text { (MG-225214), R } 250 \\
\text { (MG-228222), R } 291 \text { (MG-228263); Sa s.n. } \\
\text { (MAR-6614) }\end{array}$ & Amz, Caa, Cer & $\mathrm{Sr} / \mathrm{Sc}$ & $\mathrm{He}$ \\
\hline Xyris savanensis Miq. & Mo 3187 (MG-214816) & Amz, Caa, Cer, Atl, Pmp, Pnt & Rfi & $\mathrm{He}$ \\
\hline Xirys sp. nov. & $\begin{array}{l}\text { Mo } 2851 \text { (MG-211544), Mo } 3162 \text { (MG- } \\
\text { 214791); R } 19 \text { (MG-225182, R } 21 \text { (MG- } \\
\text { 225184), R } 51 \text { (MG-22514) }\end{array}$ & - & Il & $\mathrm{He}$ \\
\hline
\end{tabular}

The 289 recorded species are distributed in 189 genera and 73 families (Tab. 1), and the most representative families were Poaceae with 37 species (12\%), Cyperaceae 35 ssp. (12\%), Fabaceae 31 spp. (10\%), Rubiaceae 17 spp. (5\%), Eriocaulaceae ten spp. (3\%), Myrtaceae nine spp. (3\%), Apocynaceae and Polygalaceae eight spp. (2\%), Xyridaceae seven spp. (2\%) and Euphorbiaceae six spp. (2\%) (Fig. 2).

The predominant habit was herbaceous, with $53.28 \%$ of the species, followed by shrubs and trees, with $13.14 \%$ and $12.45 \%$, respectively. Representing less than $8 \%$ of the species, the lianescent habit was the least abundant in the study area (Fig. 3).

In this study, 56 new species records were made for Maranhão state (Tab. 1), including 15 species widely distributed in Brazil: Actinostachys pennula (Schizaeaceae), Calophyllum brasiliense (Calophyllaceae), Rhynchospora filiformis (Cyperaceae), Eriocaulon cinereum (Eriocaulaceae), Centrosema pascuorum (Fabaceae), Utricularia myriocista (Lentibulariaceae), Heteropterys nervosa (Malpighiaceae),
Mayaca longipes (Mayacaceae), Myrciaria floribunda (Myrtaceae), Paspalum pumilum, Trichanthecium polycomum, Sacciolepis vilvoides (Poaceae), and Spermacoce prostrata (Rubiaceae) (Flora do Brasil 2020 2018).

Two undescribed species were recorded, Mesosetum sp. nov. (Poaceae) and Xyris sp. nov. (Xyridaceae), which are undergoing description prior to publication. The only species considered threatened with extinction, in the endangered (EN) category, was Bacopa cochlearia (MMA 2014), with only one record for the PNLM, referring to an interdunal lagoon (Lagoa Azul).

\section{Description of phytophysiognomies}

The vegetation in the study area is classified here under eight distinct phytophysiognomies, occurring with most frequency in the PNLM and characterized as following:

1) Halophytic Vegetation: tidal areas with plants that have some resistance to salinity (Fig. 4A-B). In this formation we can observe herbaceous plants such 

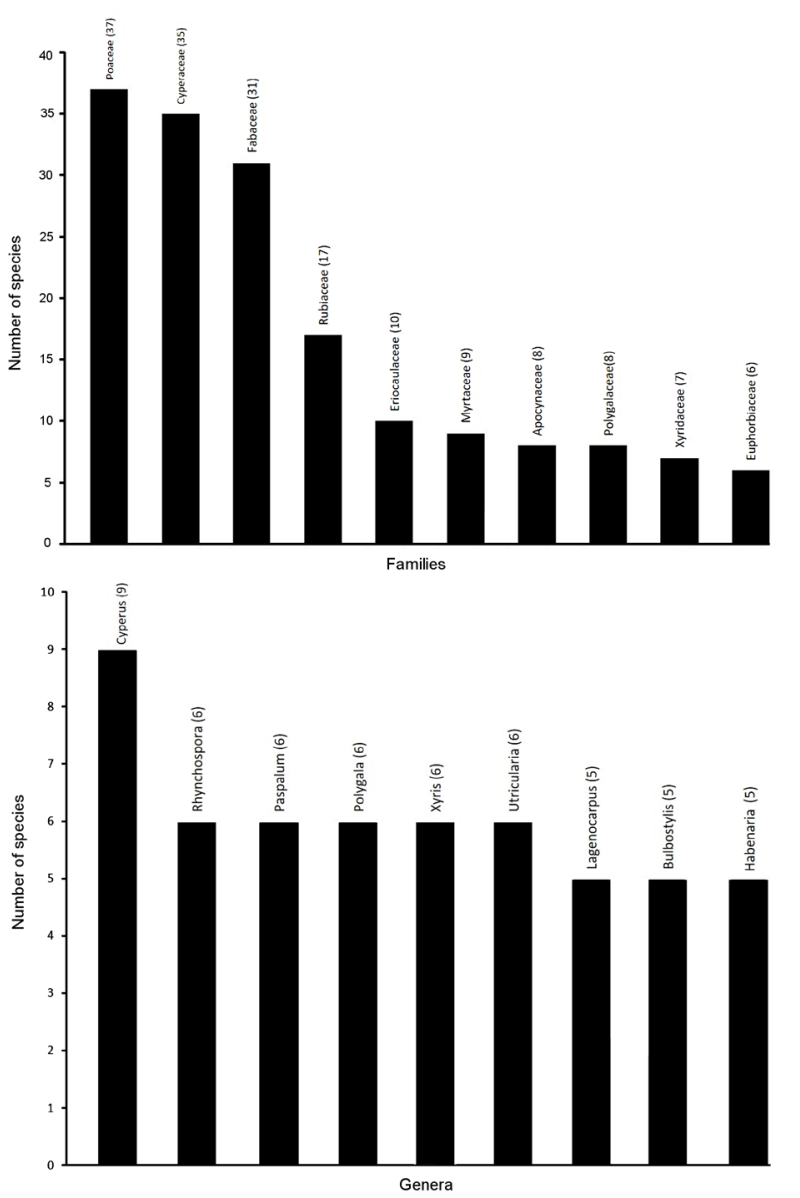

Figure 2. Richest families and genera of PNLM. Number of species for each taxon in parentheses.

as Blutaparon portulacoides (Amaranthaceae), Cyperus articulatus, C. obtusatus, C. subsquarrosus, Eleocharis geniculata (Cyperaceae), Paspalum vaginatum, Spartina alternifolia, Sporobolus virginicus (Poaceae), and sparse shrubs of Conocarpus erectus (Combretaceae);

2) Scrub: Scrub formations around dunes are generally localized on top of dunes, composed of gnarled trees and shrubs, many times with stems completely buried in sand (Fig. 4C-D). Common shrub species include Anacardium occidentale (Anacardiaceae), Chrysobalanus icaco (Chrysobalanaceae), Chamaecrista flexuosa and Indigofera microcarpa (Fabaceae), Byrsonima laevis and B. sericea (Malpighiaceae), Mouriri guianensis (Melastomataceae). Within the herbaceous species, Cyperus crassipes and Rhynchospora tenuis (Cyperaceae), Chamaecrista flexuosa and Indigofera microcarpa (Fabaceae) and Trachypogon spicatus (Poaceae) stand out;

3) Interdunal lagoons: lagoons/ ponds that form as a result of the accumulation of precipitation during the rainy period (Fig. 4E-F). These water bodies present vegetation constituted primarily by annual herbs, such as Helantium tenellum (Alismataceae), Eriocaulon cinereum, E. setaceum and Paepalanthus sessiliflorus (Eriocaulaceae), Schultesia guianensis (Gentianaceae), Utricularia cornuta and U. subulata

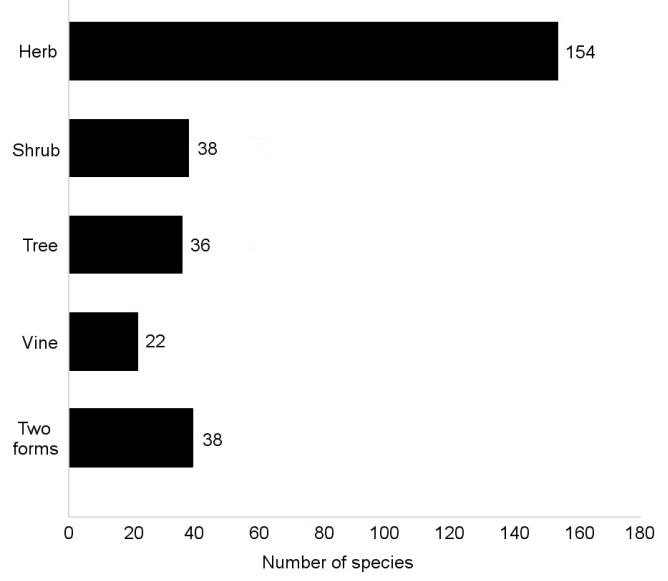

Figure 3. Habits of the species occurring in the PNLM.

(Lentibulariaceae), Bacopa cochlearia (Plantaginaceae), Xyris jupicai and Xyris sp. nov. (Xyridaceae) that appear in the margins of the lagoons at the beginning of the dry period and dominate the entire bed of the lagoon in the peak of drought, when they are completely empty. Few perennial species are observed in this environment, though Panicum aquaticum (Poaceae) and Nymphoides humboldtiana (Menyanthaceae) are generally found in the lagoons during the rainy season;

4) Restinga fields: Hydromorphic fields or sandy marshes of oligotrophic soils with mostly continuous stratum (Fig. 5A-B). During the rainy season they can become flooded and are also characterized by a large number of annual species such as Burmania capitata (Burmaniceae), Drosera sessilifolia (Droseraceae), Paepalanthus bifidus, $P$. polytrichoides and Syngonanthus cuyabenses (Eriocaulaceae), Utricularia benjaminiana and U. myriocista (Lentibulariaceae), Polygala adenophora (Polygalaceae), Habenaria spp. (Orchidaceae), Xyris paraensis (Xyridaceae), amongst others. Herbaceous perennial species are also observed, including Euploca polyphylla (Heliotropiaceae), Gomphrena sp. (Amaranthaceae), Bulbostylis conifera, B. junciformis, Cyperus haspan, Lagenocarpus spp. (Cyperaceae), Abolboda pulchella (Xyridaceae), Tetraulacium veroniciforme (Plantaginaceae). Within these, shrub species Krameria tomentosa (Krameriaceae), Cuphea antisyphilitica (Lythraceae), Comolia villosa (Melastomataceae) and Dizygostemon sp. (Plantaginaceae) stand out;

5) Shrubby Restinga: an area formed from both continuous and discontinuous ground cover, with a higher frequency of shrubs and isolated trees, also forming clumps, with physiognomy resembling Cerrado sensu strictu (Fig. 5C). Within the tree and shrub species, Himatanthus articulatus (Apocynaceae), Monteverdia erythroxyla (Celastraceae), Chrysobalanus icaco (Chrysobalanaceae), Clusia grandiflora (Clusiaceae), Humiria balsamifera (Humiriaceae), Vismia guianensis (Hypericaceae), Byrsonima sericea (Malpighiaceae), Eugenia punicifolia (Myrtaceae) 
and Ouratea caudata (Ochnaceae) stand out. Lagenocarpus guianensis (Cyperaceae), Andropogon leucostachyus, Mesosetum loliiforme, Paspalum carinatum, Streptostachys asperifolia (Poaceae) are some of the herbaceous species found in this phytophysiognomy. Lianescent species are frequently observed, such as Mandevilla hirsuta and $M$. scabra (Apocynaceae), Ipomoea spp. (Convolvulaceae), Davilla cearensis and Doliocarpus spraguei (Dilleniaceae), and Passiflora foetida (Passifloraceae);
6) Restinga forest: formed by a dense layer of trees and shrubs, producing a continuous canopy. Generally, presents individuals with thin, gnarled trunks growing on ground with accumulated organic material, and this is loose in comparison to what is seen in open restinga. Another defining characteristic of species is their pronounced deciduousness, with a xeromorphic aspect (Fig. 5D). In this formation the prominent presence of Protium heptaphyllum subsp. ulei (Burseraceae), Caryocar brasiliense
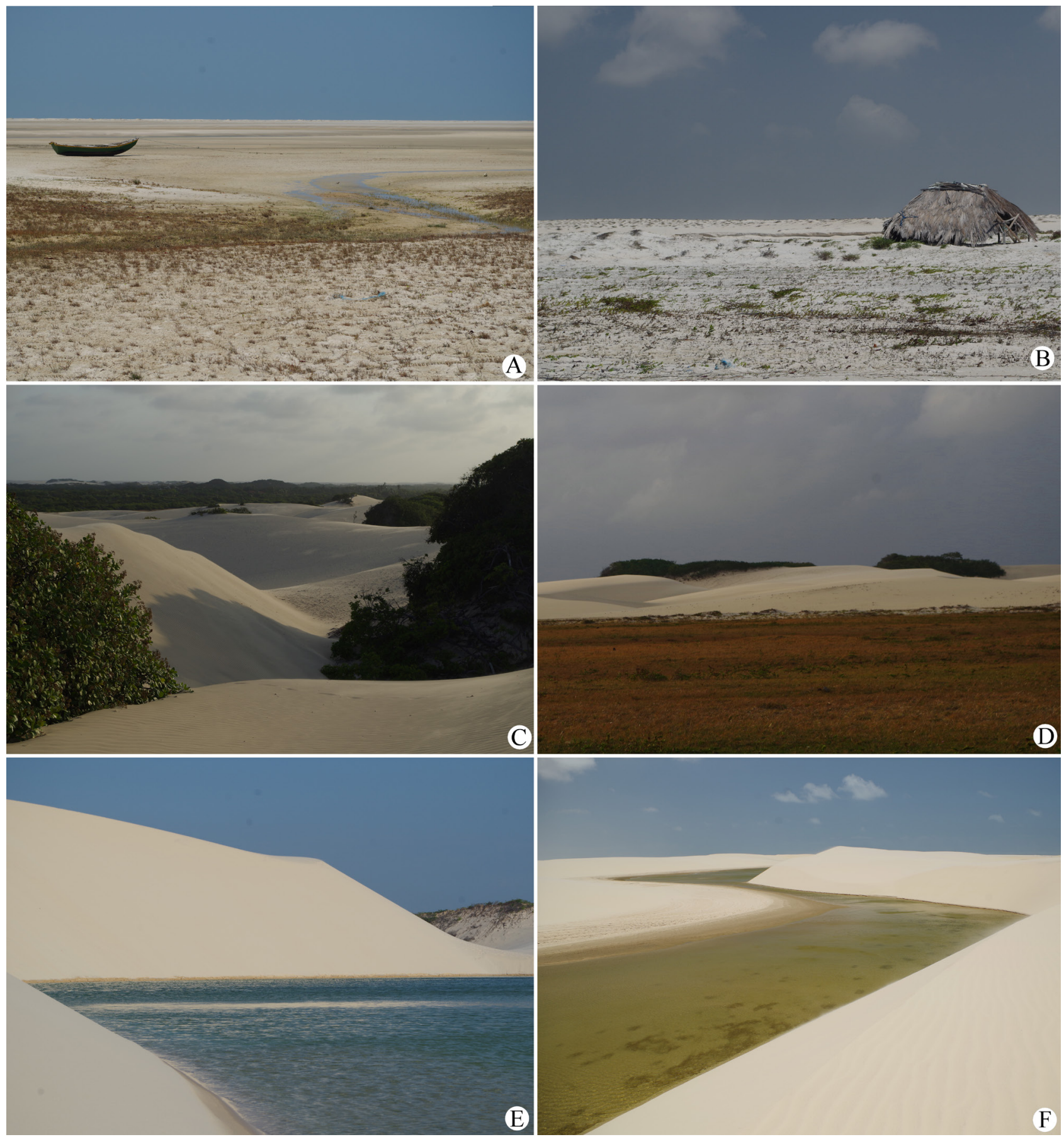

Figure 4. Main phytophysiognomies of the PNLM. A-B. Halophytic vegetation. C-D. Scrub. E-F. Interdunal Lagoons. Photographs by Pedro Viana (A-C, E-F) and Nara Mota (D). 
Vascular flora of Lençóis Maranhenses National Park, Maranhão State, Brazil: checklist, floristic affinities and phytophysiognomies of restingas in the municipality of Barreirinhas

(Caryocaraceae), Abarema cochleata, Andira vermifuga, Copaifera martii, Dimorphandra gardneriana and Hymenaea velutina (Fabaceae), Myrcia multiflora and Myrcia splendens (Myrtaceae), Matayba discolor (Sapindaceae), Manilkara triflora (Sapotaceae) are observed;

7) Cocais forest: phytophysiognomy characterized by the abundant presence of palms Copernicia prunifera, occasionally occurring sympatrically with Mauritia flexuosa.
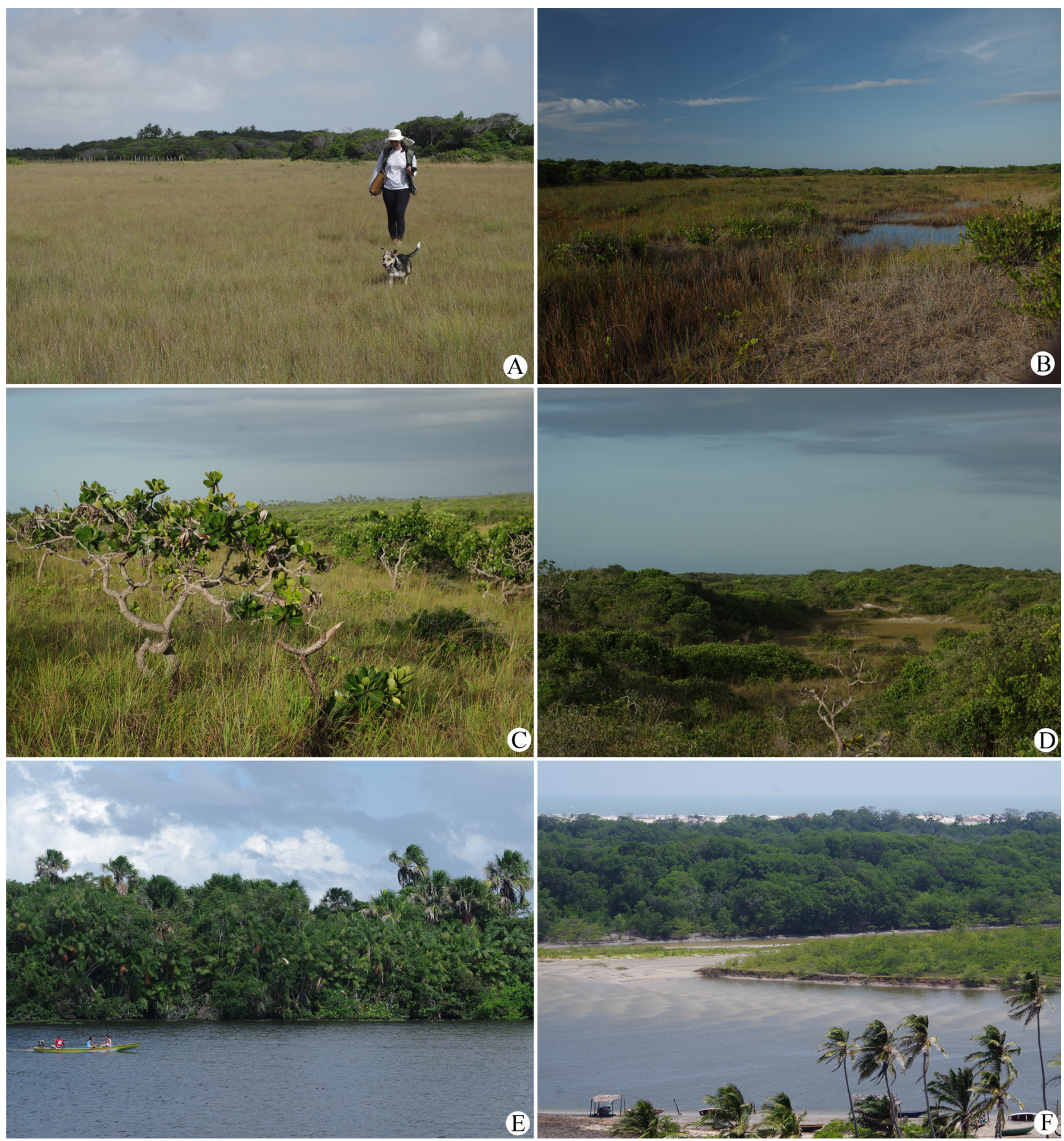

Figure 5. Main phytophysiognomies of the PNLM. A-B. Restinga fields. C. Shruby Restinga. D. Shruby Restinga and in the background restinga forest. E. Gallery Forest. F. River Preguiça mouth, showing the mangrove. Photographs by Nara Mota (A-D) and Pedro Viana (E-F).
8) Gallery forest and Mangroves: vegetation occurring in the banks of the Preguiça River and its tributaries (Fig. 5E). Proximity to the ocean determines the composition of species of this physiognomy. Close to the estuary, the vegetation is typically of mangroves with low species richness and the predominance of few tree species (Fig. $5 \mathrm{~F}$ ), such as Rhizophora harrisonii and $R$. racemosa (Rhizophoraceae). As distance from the ocean increases and salinity falls, 
the species richness increases, and species of Arecaceae, such as Mauritia flexuosa, some Annonaceae and Araceae can be observed.

\section{Floristic similarity}

The similarity indexes calculated between the areas (Tab. 2) ranged from 0.082 (Pará and Piauí states restingas) to 0.197 (this study and Pará state restingas). According to Kent \& Coker (1992), values larger than or equal to 0.5 indicate high similarity. Therefore, it can be inferred that the similarity between the areas is to be considered low.

The clustering analysis (Fig. 6) revealed a group formed between the study area and restingas of Pará state, which, in turn, is related with the restingas of Maranhão, but with low similarity (similarity index 0.178 ).

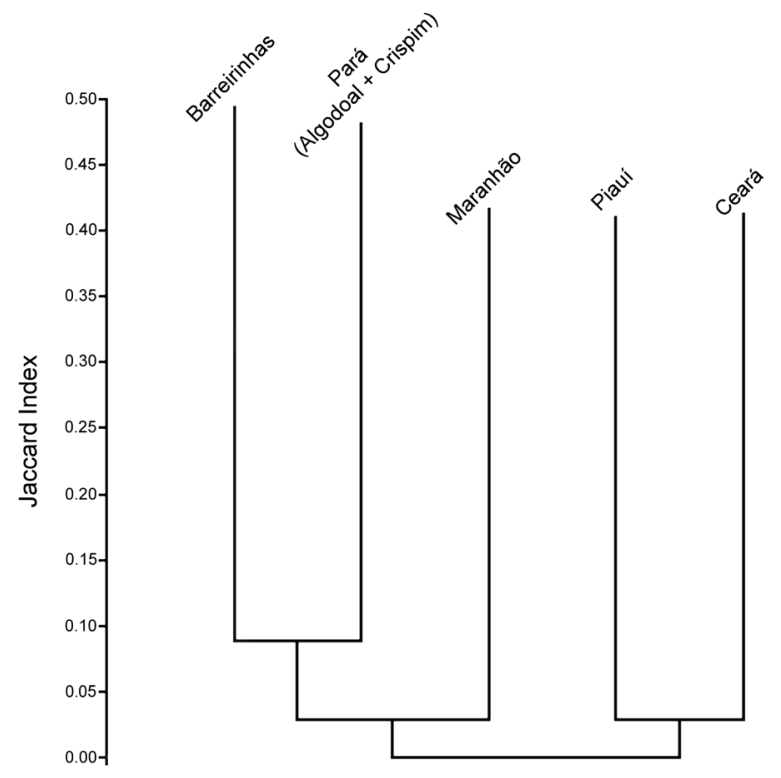

Figure 6. Dendogram of Floristic Similarity between the study areas analysed: Barreirinhas: this study; Pará state: Algodoal/ Maiandeua and Crispim/Marapanim (Amaral et al. 2008); Piauí state (Santos-Filho et al. 2015); Maranhão state (Almeida Junior et al. 2017); Ceará state (Santos-Filho et al. 2011).

\section{Discussion}

The families with higher species richness in this research coincide with the richest families for other studies of
Brazilian restingas (Araújo 2000; Pereira \& Araújo 2000; Santos et al. 2003; Almeida Júnior et al. 2007; 2017; Amaral et al. 2008; Santos-Filho et al. 2011; 2015; Gomes \& Guedes 2014; Serra et al. 2016). According to Melo Júnior \& Boeger (2015), families like Fabaceae and Myrtaceae present high numbers of species in the restinga. Amaral et al. (2008) detected Fabaceae, Poaceae and Cyperaceae as the richest families on the restinga in Pará and Amapá states.

The low number of fern species and lycophytes may be explained by the biological particularities of these groups, which are largely dependent on factors such as the climate, availability of water and substrate type (Gonzatti et al. 2014). The PNLM presents a longer period of drought, associated with sandy soil with low water retention, which can discourage the establishment of fern and lycophyte species.

Although southeastern Maranhão is considered one of the largest knowledge gaps for the understanding of the Brazilian flora (BFG 2015), the presence of 56 new records for the state within the PNLM, along with the two new species (Tab. 1), demonstrates that, in reality, the whole of Maranhão state, including the restingas (Zickel et al. 2004; Ribeiro 2011) represents a huge knowledge gap and needs careful botanical exploration. There is a need for botanical research into the different vegetal formations of Maranhão to better understand the floristic richness of the state.

The breakdown of the new records found within this work is interesting. Some were previously cited predominantly for the Amazon biome: Paepalanthus polytrichoides (Eriocaulaceae), Doliocarpus spraguei (Dilleniaceae), Leptolobium nitens, Vatairea sericea (Fabaceae), Eschweilera decolorans (Lecythidaceae), Utricularia benjaminiana (Lentibulariaceae), Borreria paraensis, Duroia paraensis (Rubiaceae), Ternstroemia delicatula (Pentaphyllaceae), Eragrostis guianensis, Reimarochloa aberrans, Rhytachne guianensis (Poaceae). Four were cited, in Brazil, for the Cerrado: Lepidaploa rufogrisea (Asteraceae), Bulbostylis lagoensis (Cyperaceae), Syngonanthus philodicoides (Eriocaulaceae), Asemeia rhodoptera (Polygalaceae). Finally, three were previously cited for the Caatinga biome: Stilpnopappus cearensis (Asteraceae), Rhynchospora curvula (Cyperaceae) and Bacopa cochlearia (Plantaginaceae). These results demonstrate how these three biomes contribute to the composition of the PNLM flora, revealing its ecotonal character (Santos-Filho et al. 2013a). In this way, different features and plant synusia typical of the mid-Northern region of Brazil can be found, accompanied by corresponding soil and climate variations (Santos-Filho et al. 2013b).

Table 2. Matrix of similarity coefficients (Jaccard) between the compared areas. Barreirinhas: this study; Pará state: Algodoal/ Maiandeua and Crispim/Marapanim (Amaral et al. 2008); Piauí state (Santos-Filho et al. 2015); Maranhão state (Almeida Junior et al. 2017); Ceará state (Santos-Filho et al. 2011).

\begin{tabular}{|c|c|c|c|c|}
\hline & Barreirinhas & Pará state & Piaui state \\
\hline Pará state & 0.19789474 & 1 & 1 \\
\hline Piauí state & 0.093587522 & 0.082236842 & 0.18167457 \\
\hline Maranhão state & 0.12457338 & 0.17869416 & 0.23278689 \\
\hline Ceará state & 0.10869565 & 0.10754414 & 0.15727003 \\
\hline
\end{tabular}


In relation to the species habit, the data was found to be similar to other flora studies of restingas in Northern and Northeastern Brazil (e.g. Amaral et al. 2008; Santos-Filho et al. 2015; Oliveira et al. 2015), with the predominance of herbaceous species, followed by shrub and tree species, reinforcing the vegetation classification of the region (sensu IBGE 2012) as pioneer formations, with a higher number of species adapted to survive in adverse situation (Oliveira et al. 2015). According to Martins \& Batalha (2011) herbs, in general, present the best resistence to drought, withstanding high luminosity and strong winds, making it possible for them to survive in different restinga physiognomies in the PNLM. The high similarity between the restinga flora in PNLM Barreirinhas and the restingas in Pará state suggests the colonization of coastal areas of Maranhão from the Amazon biome. These facts are also corroborated by the new records the PNLM flora, where the Amazonian Rainforest contributes with the largest number of exclusive species (12 spp.), when compared with the Cerrado and Caatinga (four species each).

The similarity of PNLM flora with the areas compared in this study is considered low. This panorama can be explained by the location of PNLM, which is an area of transition between three biomes. In the study area they were recorded as species from the Amazon, Caatinga and Cerrado, revealing a unique character towards its flora. Compared with areas of restinga found exclusively in the Caatinga or Amazon biomes, the similarity of PNLM flora is clearly demonstrated to be low. Nevertheless, these results could be influenced by eventual discrepancies in the sampling efforts utilized in the different studies.

Based on these results it may be concluded that the restingas of the PNLM in the municipality of Barreirinhas present a high number of species, including many that have not been previously recorded from Maranhão. This high number of new records highlights the necessity to increase collections in Maranhão state, including all its plant formations. The peculiarity of the flora in the study area was revealed through the occurrence of new species. The low similarity with other areas of restinga studied justifies the importance of continuing floristic studies in the PNLM, especially in areas not covered by the present study.

\section{Acknowledgements}

Thanks to the Museu Paraense Emílio Goeldi and the Universidade Federal Rural da Amazônia; ICMBio in Barreirinhas; the curators of the IAN (EMBRAPA), MAR (Universidade Federal do Maranhão) and MG (Museu Paraense Emílio Goeldi) herbaria; the specialists identifiers and Temístocles Rodrigues. We thank Vanessa Zappi-Taylor and Daniela C. Zappi for the English version. This study received financial support from the CAPES no. PROAmazônia 3268/2013.

\section{References}

Almeida Júnior EB, Pimentel RMM, Zickel CS. 2007. Flora e formas de vida em uma área de Restinga no litoral norte de Pernambuco. Revista de Geografia 24: 19-34.

Almeida Júnior EB, Silva ANF, Lima GP, et al. 2017. Checklist of the flora of th restingas of Maranhão State, Northeast, Brazil. India Journal of Applied Research 7: 603-612.

Amaral DD, Prost MT, Bastos MNC, Costa Neto SV, Santos JUM. 2008. Restingas do litoral amazônico, estados do Pará e Amapá, Brasil. Boletim do Museu Paraense Emílio Goeldi Ciências Naturais 3: 35-67.

APG - Angiosperm Phylogeny Group IV. 2016. An update of the Angiosperm Phylogeny Group classification for the orders and families of flowering plants: APG IV. Botanical Journal of the Linnean Society 181: 1-20.

Aragão JG, Conceição GM. 2008. Myrtaceae: espécies das subtribos Eugeniinae, Myrciinae e Myrtiinae registradas para o estado do Maranhão. Revista Sinapse Ambiental 1: 7-17.

Araújo DSD. 2000. Análise florística e fitogeográfica das restingas do Estado do Rio de Janeiro. PhD Thesis, Universidade Federal do Rio de Janeiro, Rio de Janeiro.

Araújo ACM, Silva ANF, Almeida Júnior EB. 2016. Caracterização estrutural e status de conservação do estrato herbáceo de dunas da Praia de São Marcos, Maranhão, Brasil. Acta Amazonica 46: 247-258.

Bandeira ICN. 2013. Geodiversidade do estado do Maranhão. Teresina, CPRM.

Bastos MNC. 1995. A Importância das formações vegetais da restinga e do manguezal para as comunidades pesqueiras. Boletim do Museu Paraense Emílio Goeldi Série Antropologia 11: 41-56.

Bastos MNC, Costa DCT, Santos JUM. 2003. Vegetação de Restinga: Aspectos botânicos e uso medicinal. Canadá, Museu Paraense Emílio Goeldi, Projeto Renas/IDRC/CRDI.

BFG - The Brazil Flora Group. 2015. Growing knowledge: an overview of seed plant diversity in Brazil. Rodriguésia 66: 1085-1113.

BWG - Boraginales Working Group. 2016. Familial classification of the Boraginales. Taxon 63: 502-522.

Castro ACL, Piorski NM. 2002. Plano de Manejo do Parque Nacional dos Lençóis Maranhenses. São Luís, Fundação Sousândrade de Apoio e Desenvolvimento da Universidade Federal do Maranhão, Laboratório de Hidrologia.

CNCFLORA - Centro Nacional de Conservação da Flora. 2018. Lista vermelha. http://cncflora.jbrj.gov.br/portal/pt-br/listavermelha. 9 Mar. 2018.

Damme K, Dumont H.J. 2010. Cladocera of the Lençóis Maranhenses (NE - Brazil): Faunal composition and a reappraisal of Sars' Method. Brazilian Journal of Biology 70: 775-779.

Flora do Brasil 2020 em construção. 2018. Flora do Brasil 2020. Jardim Botânico do Rio de Janeiro. http://floradobrasil.jbrj.gov.br/. 17 Sep. 2018.

Fidalgo O, Bononi VLR. 1989. Técnicas de coleta, preservação e herborização de material botânico. São Paulo, Instituto de Botânica.

Gomes FS, Guedes MLS. 2014. Flora vascular e formas de vida das formações de restinga do litoral norte da Bahia, Brasil. Acta Biológica Catarinense 1: 22-43.

Gonzatti F, Valduga E, Wasum RA, Scur L. 2014. Florística e aspectos ecológicos de licófitas e samambaias do litoral médio do Rio Grande do Sul, Brasil. Revista Brasileira Biociências 12: 215-225.

IBGE - Instituto Brasileiro de Geografia e Estatística. 2012. Manual técnico da vegetação brasileira. 2nd. edn. Rio de Janeiro, Fundação do Instituto Brasileiro de Geografia e Estatística.

ICMBIO. 2016. Parque Nacional dos Lençóis Maranhenses. Barreirinhas, ICMBio - MMA. http://www.icmbio.gov.br/parnalencoismaranhenses/ quem-somos.html. 6 Jun. 2016.

Kent M, Coker P. 1992. Vegetation description analyses. London, Behaven Press. Kramer KU, Green PS. 1990. The families and genera of vascular plants. Volume 1. Pteridophytes and gymnosperms. Berlin, Springer-Verlag.

Lima GP, Almeida Júnior EB. 2018. Diversidade e similaridade florística de uma Restinga ecotonal no Maranhão, Nordeste do Brasil. Revista Interciencia 43: 275- 282. 


\section{Misael Lira Rodrigues, Nara Furtado de Oliveira Mota, Pedro Lage Viana, Ana Kelly Koch and Ricardo de S. Secco}

Maranhão. 2002. Atlas do Maranhão gerenciamento e desenvolvimento econômico. São Luís, GEPLAN.

Martins FR, Batalha MA. 2011. Formas de vida, espectro biológico de Raunkiaer e fisionomia da vegetação. In: Felfili JM, Eisenlohr PV, Melo MMRF, Andrade LA, Meira-Neto JAA. (eds.). Fitossociologia no Brasil: métodos e estudos de casos. Viçosa, Editora UFV. p. 44-85.

Melo Júnior JCF, Boeger MRT. 2015. Riqueza, estrutura e interações edáficas em um gradiente de restinga do Parque Estadual do Acaraí, Estado de Santa Catarina, Brasil. Hoehnea 42: 207-232.

Miranda JP, Costa JCL, Rocha CFD. 2012. Reptiles from Lençóis Maranhenses National Park, Maranhão, northeastern Brazil. Zookeys 246: 51-68.

MMA - Ministério do Meio Ambiente. 2014. Portaria MMA no 443, de 17 de dezembro de 2014. Lista nacional oficial de espécies da flora ameaçadas de extinção. http://cncflora.jbrj.gov.br/portal/static/pdf/ portaria_mma_443_2014.pdf

Moschini-Carlos V, Pereira D, Wisniewski MSJ, Pompêo MLM. 2008. The planktonic community in tropical interdunal ponds (Lençóis Maranhenses National Park, Maranhão State, Brazil). Acta Limnologica Brasiliensia 20: 99-110.

Moschini-Carlos V, Pompêo MLM. 2016. Características Gerais da Região do Parque Nacional dos Lençóis Maranhenses, Maranhão, Brasil. USP. http://ecologia.ib.usp.br/portal/index.php?option=com content\&view=article\&id=71\&Itemid=410. 6 Aug. 2016 .

Oliveira EVS, Ferreira Sobrinho ES, Landim MF. 2015. Flora from the restingas of Santa Isabel Biological Reserve, northern coast of Sergipe state, Brazil. Check List 11: 1-10.

Pereira OJ, Araujo DSD. 2000. Análise florística das restingas dos estados do Espírito Santo e Rio de Janeiro. In: Esteves FA, Lacerda LD. (eds.) Ecologia de restingas e lagoas costeiras. Macaé, Nupem/Universidade Federal do Rio de Janeiro. p. 25-63.

Ribeiro EKMD. 2011. Fenologia e atributos reprodutivos de espécies ocorrentes em restingas no Maranhão. PhD Thesis, Universidade Federal de Pernambuco, Recife.

Rothfels CJ, Sundue MA, Larsson Li-YKA, Kato M, Schuettpelz E, Pryer KM. 2012. A revised family-level classification for eupolypod II ferns (Polypodiidae: Polypodiales). Taxon 61: 515-533.
Santos JUM, Amaral DD, Gorayeb IS, et al. 2003. Vegetação da Área de Proteção Ambiental Jabotituia-Jatuim. Município de Viseu, Pará, Brasil. Acta Amazonica 33: 431-444.

Santos-Filho FS, Almeida Júnior EB, Bezerra LFM, Lima LF, Zickel CS. 2011. Magnoliophyta, restinga vegetation, state of Ceará, Brazil. Check List 7: 478-485.

Santos-Filho FS, Almeida Júnior EB, Lima PB, Soares CJRS. 2015. Checklist of the flora of the restingas of Piauí state, Northeast Brazil. Check List 11: 1-10.

Santos-Filho FS, Almeida Júnior EB, Soares CJR. 2013a. Cocais: Zona ecotonal natural ou artificial? Revista Equador 1: 2-13.

Santos-Filho FS, Almeida Júnior EB, Zickel CS. 2013b. Do edaphic aspects alter vegetation structures in the Brazilian restinga? Acta Botanica Brasilica 27: 613-623.

Saraiva NA, Fernandes Pinto E. 2007. Extrativismo, economia solidária e desenvolvimento sustentável na região dos Lençóis Maranhenses. São Paulo, USP.

Serra FCV, Lima PB, Almeida Júnior EB. 2016. Species richness in restinga vegetation on the eastern Maranhão State, Northeastern Brazil. Acta Amazonica 46: 271-280.

Smith AR, Pryer KM, Schuettpelz E, Korall P, Schneider H, Wolf PG. 2006. A classification for extant ferns. Taxon 55: 705-731.

Sneath PH, Sokal RR. 1973. Numerical taxonomy the principles and practice of numerical classification. San Francisco, W.H. Freeman and Company.

Thiers B. 2009. [continuamente atualizado]. Index Herbariorum: A global directory of public herbaria and associated staff. New York Botanical Garden's Virtual Herbarium. http://sweetgum.nybg.org/ih/. 13 Jan. 2018.

Walter BMT, Fagg CW. 2015. Coleta, preparo e documentação de material botânico testemunho. In: Eisenlohr PV, Felfili JM, Melo MMRF, Andrade LA, Meira Neto JAA. (eds.) Fitossociologia no Brasil: Métodos e estudos de casos. Viçosa, Editora da Universidade Federal de Viçosa. p.13-30.

Zickel CS, Vicente A, Almeida Júnior EB, Cantarelli JRR, Sacramento AC. 2004. Flora e vegetação das restingas no Nordeste Brasileiro. In: Eskinazi-Leça E, Neumann-Leitão S, Costa MF. (eds.) Oceanografia: um cenário tropical. Recife, Bargaço. p. 689-701. 